\title{
Review on the advancements of magnetic gels: towards multifunctional magnetic liposome-hydrogel composites for biomedical applications
}

\author{
Sérgio R. S. Veloso, ${ }^{a}$ Raquel G. D. Andrade, ${ }^{a}$ and Elisabete M. S. Castanheira ${ }^{a, *}$ \\ ${ }^{a}$ Centre of Physics, University of Minho, Campus de Gualtar, 4710-057 Braga, Portugal \\ *ecoutinho@fisica.uminho.pt
}

\begin{abstract}
Magnetic gels have been gaining great attention in nanomedicine, as they combine features of hydrogels and magnetic nanoparticles into a single system. The incorporation of liposomes in magnetic gels further leads to a more robust multifunctional system enabling more functions and spatiotemporal control required for biomedical applications, which includes on-demand drug release. In this review, magnetic gels components are initially introduced, as well as an overview of advancements on the development, tuneability, manipulation and application of these materials. After a discussion of the advantages of combining hydrogels with liposomes, the properties, fabrication strategies and applications of magnetic liposome-hydrogel composites (magnetic lipogels or magnetolipogels) are reviewed. Overall, the progress of magnetic gels towards smart multifunctional materials are emphasized, considering the contributions for future developments.
\end{abstract}

\section{Introduction}

Over the last decades, significant advances have been made in cancer therapy and theranostics, i.e. the combined use of therapeutic and diagnostic agents. These signs of progress are a result of nanomedicine multidisciplinary efforts towards the development of smart multifunctional nanomaterials. Smart materials offer the possibility of manipulating their properties upon an external stimulus, opening new paths for strategies towards drug delivery [1], cell differentiation [2], transmission of information [3], and improvements of contrast agents [4].

An important category of soft smart materials is the magnetogels (also called magnetic hydrogels/gels or ferrogels), which consist of magnetic nanoparticles embedded in an elastic matrix $[5,6]$. These materials belong to the family of magnetic colloids that are divided into two main groups: the (I) magnetic suspensions and (II) magneto-polymers [7]. The magnetic suspensions encompass the ferrofluids and magnetorheological suspensions, which differ on the nanoparticle size. The former contains particles less than $50 \mathrm{~nm}$ and the latter is composed of micron- or submicron-sized particles [7,8]. The magneto-polymers consist of magnetic nanoparticles dispersed in an elastic dry polymer network, the elastomers, or in a soft viscoelastic gel (water or organic solvent), the magnetic gels.

The presence of magnetic nanoparticles allows real-time remote control over the micro- and macroscopic properties, such as the shape, size, drug diffusion and mechanical behaviour [6,9]. Further, the nanoparticles can modify the hydrogel network structure through non-covalent or covalent bonding, which offers control over the properties of the pristine material. This means that the adsorption, mechanical, electrical, optical and thermal properties can be modulated $[6,10,11]$, but depend on various parameters. Such parameters include (I) concentration, composition, size and shape of the nanoparticles; (II) length, chemical composition and concentration of the hydrogel network chains; (III) cross-linking degree of the network; (IV) the density of nucleation sites induced by the nanoparticles, that might affect the drug loading efficiency $[12,13] ;(V)$ the distribution of the nanoparticles within the hydrogel network; (VI) the type of interactions between the nanoparticles and the hydrogel fibers (physical or chemical); (VII) the swelling degree of the network; (VIII) and the method of preparation of the magnetic gel $[7,9,14]$.
The magnetic gels endow the remarkable property that they can not only be shaped like their counterparts (hydrogels), but can also be categorized into isotropic or anisotropic, which offers new and improved possibilities in biomedical applications. An example of anisotropic magnetogels (figure 1) was developed by ContrerasMontoya and coworkers [9] using PEG (polyethylene glycol) coated magnetic nanoparticles embedded in a supramolecular hydrogel.

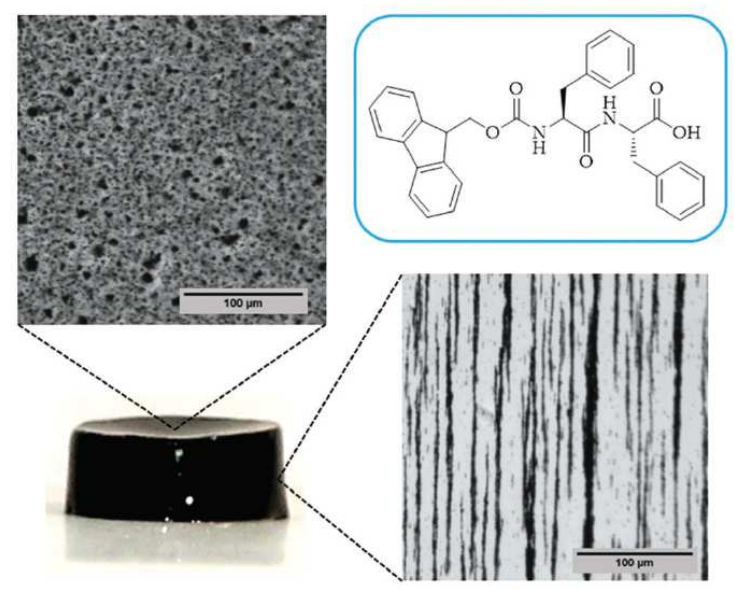

Figure 1. Macroscopic picture of a supramolecular anisotropic magnetogel, chemical structure of the hydrogelator and optical image of the top and side view. Reprinted from [9] with permission from Royal Society of Chemistry, 2020.

These ongoing innovations of magnetic gels makes them promising materials for various applications, such as controlled cell growth [15], depollution [16,17], actuators [18], biosensors [19], catalysis [20,21], microfluidic valves [22], contrast agents [23], photothermia [24], magnetic hyperthermia [25], and controlled drug delivery [26]. For more information on the biomedical applications of magnetogels, references [6], [27] and [28] are recommended.

The control of drug release remains a challenge and it is a current trend required for clinical translation of nanomedicines [29]. A recently developed mixed composite system that can tackle this challenge is the combination of liposomes with hydrogels [30], which becomes even more robust with the incorporation of 
magnetic nanoparticles (possibly named "magnetolipogels" or magnetic liposome-hydrogels). Hereby, this review seeks to answer the questions "How can magnetic liposome-hydrogel systems be developed?" and "How can it benefit the control over drug release?". Considering these two points, the reader is firstly introduced to the concepts of the two major components (hydrogels and magnetic nanoparticles). Then, the physical properties of magnetic gels and modulation of drug release through a combination of hydrogels and liposomes are highlighted. The final sections include a review and critical assessment of the developments of magnetic lipogels, its advantages, and applications.

\section{Overview of the components}

\subsection{Magnetic nanoparticles}

Magnetic materials are classified according to their response to an external magnetic field [31,32]. For instance, diamagnetic materials weakly repeal the external magnetic field (negative magnetic susceptibility), while paramagnetic materials align with the external magnetic field, but have no remanence magnetization. The positive long-range ordering exchange interactions leads to parallellyaligned magnetic moments in ferromagnetic materials, displaying magnetic domains that align under a magnetic field and enhance the magnetic flux density (positive magnetic susceptibility). Antiferromagnetic materials have antiparallelly-oriented magnetic moments of equal magnitude, while in the ferrimagnetic materials, the opposite magnetic moments have unequal magnitude.

Besides the exchange energy and magnetocrystalline anisotropy, the existence of magnetic domains divided by Bloch walls in ferromagnetic materials is associated with the magnetostatic energy that seeks to eliminate the magnetization of the material [33]. This magnetostatic energy is proportional to particle volume, while the energy required for Bloch wall formation is proportional to the wall-domain interface area. Thus, below a critical radius it is more favorable to support the magnetostatic cost, resulting in single-domain nanoparticles (Figure 2A) [34]. In this regime, particles are uniformly magnetized and display high coercivity (a measure of the materials resistance to become demagnetized) that is maximum at the single-domain critical radius, while in the multidomain region it decreases with the materials size due to subdivision in domains. Further, unlike bulk materials, which magnetization under an applied magnetic field occurs through Bloch wall motion, in the single-domain regime it occurs through spin reversal (Néel relaxation) and/or particle motion (Brownian relaxation) (Figure 2B) [32].

Further, in magnetic gels, the Brownian mechanism is highly important as it provides a means for direct coupling between the orientation of the magnetic moments and the hydrogel network (when chemically linked), i.e. a torque exerted by an external field can get transferred onto the matrix [35].

The magnetic behaviour of a uniaxial single-domain nanoparticle can be approximated by the Stoner-Wohlfarth model and the associated energy to rotate along an easy axis can be expressed by the anisotropic energy $\left(\boldsymbol{E}_{\boldsymbol{a}}\right)$ and Zeeman energy $\left(\boldsymbol{E}_{\boldsymbol{Z}}\right)$ :

$$
E=E_{a}+E_{z}=K_{T} V \sin ^{2} \theta-H V M_{S} \cos (\theta-\Phi)
$$

In the first term (anisotropy energy), $\mathbf{K}_{\mathbf{T}}$ corresponds to the total anisotropy constant, $\mathbf{V}$ to the particle volume, $\boldsymbol{\theta}$ to the angle between the magnetization direction of the particle and the easy magnetization axis ( 0 or $\pi$ radians). In the second term, $\mathbf{H}$ is the applied magnetic field and $\boldsymbol{\Phi}$ is the angle between the easy axis and the applied magnetic field.
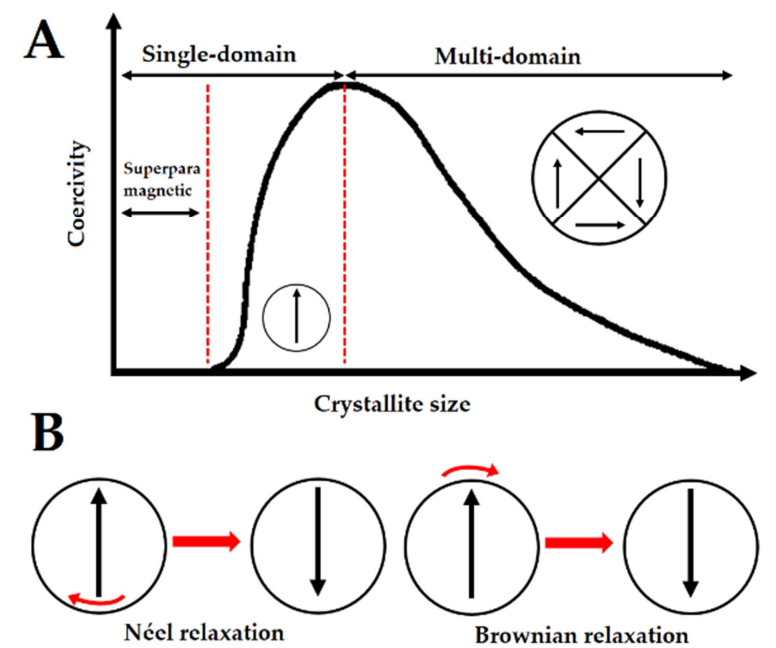

Figure 2. Schematic representation of: $(A)$ coercivity dependence on the crystallite size; (B) relaxation mechanisms in single domain nanoparticles.

Further reduction of the particle volume leads the energy required to invert the magnetic moment along the easy axis to become comparable to the thermal energy. Thus, free rotation of the magnetic moment can occur, i.e. the nanoparticle is superparamagnetic, that is characterized by the absence of coercivity and hysteresis (only displaying magnetization under an externally applied magnetic field) [34,36].

Upon application of a homogeneous static external magnetic field, the degree of alignment of non-interacting magnetic dipoles depends, on a first approximation, on the field strength and follows the Langevin law for paramagnetic substances [37]:

$$
\mathbf{M}(\boldsymbol{\alpha})=\mathbf{M}_{\mathbf{S}} \mathbf{L}(\boldsymbol{\alpha})
$$

Here, $M_{S}$ is the saturation magnetization, $L(\alpha)$ is the Langevin function, $\mathrm{L}(\alpha)=\left(\operatorname{coth} \alpha-\frac{1}{\alpha}\right)$, and $\alpha$ is the Langevin parameter, $\alpha=\frac{\mu_{0} m H}{k_{B} T}$, where $m$ is the particle magnetic moment, $\mu_{0}$ is the magnetic permeability in the vacuum, $\mathrm{k}_{\mathrm{B}}$ the Boltzmann constant, $T$ is the temperature and $H$ is the applied magnetic field.

However, the magnetization is generally increased for interacting magnetic dipoles, particularly for intermediate external fields, due to the addition of the surrounding particles field in addition to the external field. Here, a first order mean field approach can be attained assuming a Langevin-type response of the "surrounding" magnetic particles, which depends on the system's dimension (included in the parameter $\boldsymbol{c}$ ) and the density of magnetic particles [35]:

$$
\mathbf{M}(\alpha)=\mathbf{M}_{\mathbf{S}} \mathbf{L}(\boldsymbol{\alpha}+\mathbf{c L}(\alpha))
$$

Besides the orientation along the field, the external magnetic field can lead to the assembly of the nanoparticles in clusters, such as chains, that might affect the magnetic properties.

\subsubsection{Development of magnetic nanoparticles}

Currently, magnetic nanoparticles can be synthesized by different methods, either through a top-down or bottom-up approach, which encompass physical, chemical and biological methods [38]. Common physical methods include the pulsed laser ablation and 
pyrolysis, while as chemical methods the co-precipitation, solvothermal, thermal decomposition and template-assisted strategies have been thoroughly explored [39-49].

Iron oxide nanoparticles have been of major interest in cancer therapy and theranostics owing to their unique properties, such as high magnetization and chemical stability [50,51]. The ferrites magnetic moment is associated with the iron ions ordered in a ferrimagnetic spinel structure (Figure 3), which symmetry breaks at the surface of the nanoparticle [52]. The adjustment of $\mathrm{M}^{2+}$ ions chemical identity provides a means to modulate the magnetic properties. Here, the divalent cations occupy the large octahedral sites, while the small tetrahedral sites are occupied by the trivalent ions. However, $\mathrm{Zn}^{2+}$ is an exception that displays preference for the tetrahedral sites [53].

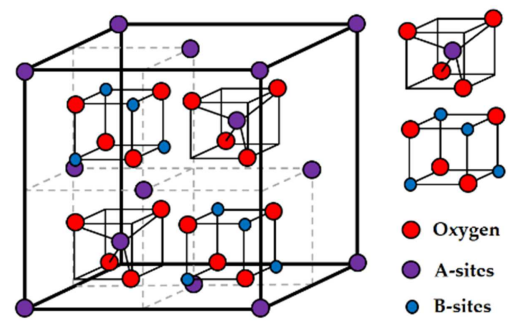

Figure 3. Schematic representation of four octants of the spinel ferrite structure. The small black cubes are also included in the back half of the unit cell. The unit cell structure consists of 32 cubic-closed pack $\mathrm{O}^{2-}$ anions and 24 metal cations structured in 2 sub-lattices antiferromagnetically coupled. These metal cations can occupy 8 of the 64 tetrahedral sites (Asites) and 16 of the 32 octahedral sites (B-sites).

Regarding the modulation of magnetic properties, the magnetic dipole moment can be roughly estimated considering $\left|\boldsymbol{m}_{N P}\right|=\boldsymbol{M V}$, where $\boldsymbol{M}$ is the total magnetization and $\boldsymbol{V}$ the particle volume [54]. In an inverse spinel, $\mathrm{Fe}^{3+}$ contribution cancels out and only divalent atoms contribute to the magnetization. However, this estimation neglects spin-orbit interactions, the position of the iron ions, size and shape of magnetic core, and surface functionalization [52].

On the development of magnetic gels, a stable colloidal solution is required to avert the irreversible agglomeration and subsequent sedimentation, leading to inhomogeneous gels. The main driving force for aggregation is the long-range dipole-dipole interactions [55], which are discussed in section 2.1.2. The total interaction energy can be expressed as the sum of the attractive magnetic dipole-dipole and van der Waals energies and of the repulsive electrostatic and steric energies. The van der Waals interactions arise from the electromagnetic fluctuations within materials $[56,57]$, whereas the electrostatic interactions are associated with the presence of surface charge and depend on the surface potential, dielectric constant of the medium, ionic strength and $\mathrm{pH}$ $[41,58]$.

The steric repulsion acts at short distances and can be achieved through the use of macromolecules or polymers (e.g. poly(ethylene glycol) - PEG) adsorbed or grafted in the nanoparticles, and arises from the mixing and elastic contributions. The former is associated with the overlapping of adjacent layers, which is repulsive only if the polymer is soluble in a solvent, while the latter is always repulsive and results from reduction of entropy when two layers approach each other [41]. Another strategy to surpass the magnetic dipole-dipole and van der Waals attraction includes the electrostatic repulsion, which consists on the use of charged macromolecules, such as charged phospholipids in the lipid bilayer of magnetic liposomes.
Usually, magnetic nanoparticles in ferrofluids are stericallycoated with surfactants (e.g. oleic acid) and suspended in a non-polar solvent [59-62]. However, in the development of magnetogels for biomedical applications, nanoparticles should be stable in water, which can be achieved with different polymers such as PEG, polyethylenimine (PEI), dextran, peptides and liposomes [63-66]. Another commonly used polymer is poly(acrylic acid) $[67,68]$, though it has been reported to negatively affect the elastic properties of supramolecular hydrogels based on dehydrodipeptides [69]. An alternative to the use of polymers is the stabilization with small molecules, such as citric acid or amino acids [70-72]. The reader is referred to references [40] and [73] for more information on the advancements of nanoparticles in bmedical applications.

\subsubsection{Relevance of magnetic dipole-dipole interactions}

In concentrated magnetic nanoparticles suspensions, besides the anisotropy and Zeeman energy terms, the dipole-dipole interactions also have to be considered $[55,74]$, as the interparticle interactions have implications on the properties (e.g. blocking temperature and reduced remanence) [75] and applications such as hyperthermia [76-78], magnetic particle imaging [52] and rheological properties [79-81]. The dipole-dipole pointlike interaction can be described as $[82,83]$ :

$$
U_{D D}=-\frac{\mu_{0}}{4 \pi}\left[3 \frac{\left(\overrightarrow{\boldsymbol{m}}_{i} \cdot \overrightarrow{\boldsymbol{r}}_{i j}\right)\left(\overrightarrow{\boldsymbol{m}}_{j} \cdot \overrightarrow{\boldsymbol{r}}_{i j}\right)}{\left|\boldsymbol{r}_{i j}\right|^{5}}+\frac{\overrightarrow{\boldsymbol{m}}_{i} \cdot \overrightarrow{\boldsymbol{m}}_{j}}{\left|\boldsymbol{r}_{i j}\right|^{3}}\right]
$$

where $\boldsymbol{r}_{i j}$ is the distance between the particle's centre at position $i$ and $j$ connected by the vector $\overrightarrow{\boldsymbol{r}}_{\boldsymbol{i j}}$, and $\mu_{\mathbf{0}}$ is the vacuum magnetic permittivity. Here, the global minimum occurs when the magnetic dipoles are displayed in a head-to-tail configuration, i.e. the dipole moment and the vector connecting the dipoles are co-aligned.

Simulation of the interactions between magnetic nanoparticles includes the use of nanoparticle models, such as soft or hard spheres. The pointlike dipole interaction can be used as an approximation of the interaction between particles, though it has to be extended to consider the coupling between soft particles under a magnetic field, as the magnetization of the adjacent particle influences the force (attractive or repulsive) between the particles [84]. Further, soft sphere potentials for the excluded volume of the particle are commonly used in molecular dynamics, such as the repulsive Weeks-Chandler-Andersen potential, that does not impose a strict bound to the minimum centre-to-centre distance (the distance is a result of the balancing of the relevant forces) [8587], and is of high importance in the study of magnetic gels/ferrogels [88-90]. On the other hand, the hard spheres are commonly used in Monte Carlo simulations, to evaluate the ground state configurations of assembled nanoparticle structures $[87,91,92]$. Further, these models have been used to study the phase diagram of assembled nanoparticles in a wide range of concentrations [93,94].

In the absence of a magnetic field, magnetic nanoparticles dipoledipole interactions can lead to the self-assembly into linear and branched chains, multi-loops, cage-like and rings structures $[86,91]$. The control of these self-assembled structures in a bottom-up approach is highly important in tuning and controlling the properties of magnetic gels, considering that these materials are usually formed through the filling of the gel matrix with the magnetic colloidal dispersion. For instance, both the assembly of the nanoparticles and magnetic response could be controlled 
through the linking mechanism, which shall display a high impact on the gel properties at high temperatures, as the dipolar chain selfassembly is suppressed by the thermal fluctuations [85]. Further, this balancing of chemical and magnetic interactions that drive the assembly of nanoparticle in superlattices can be changed through variation of the magnetic nanoparticle core size and spacing (e.g. shell, bonding motifs and degree of multivalence of the binding group) $[95,96]$.

Besides the already mentioned parameters and the use of anisotropic shaped nanoparticles [41], Janus particles offer different ground state structures depending on the orientation of the magnetic moment and the shift from the particle' centre. For instance, zipper-like structures are obtained for magnetic moments, oriented perpendicular to the radius and with small shifts $[82,87]$, which can be explored in the development of magnetic gels. For example, Yuet et al. [97] developed monodisperse superparamagnetic magnetic gel particles through a microfluidicbased synthesis, that comprise poly(ethylene glycol)-diacrylate and magnetite nanoparticles $(\sim 10 \mathrm{~nm})$. Besides the structures formed under an externally applied magnetic field (Figure 4), these materials allow the encapsulation and delivery of biologically-active compounds and the differential functionalization of the surfaces [97].

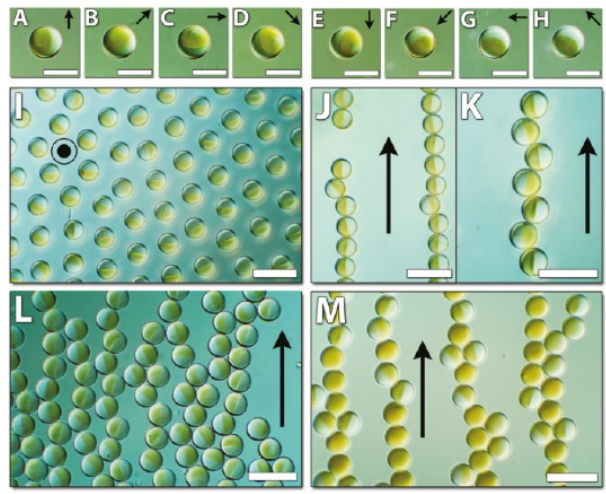

Figure 4. Differential interference contrast (DIC) images of the Janus particles in response to an external magnetic field: $(\mathrm{A}-\mathrm{H})$ rotating; (I) outof-plane; $(J, K)$ in-plane; $(L)$ in-plane at high concentration; $(M)$ 1:1 mixture of Janus and homogeneous magnetic gel particles. Scale bars are (A-H) 50 $\mu \mathrm{m}$ and (I-M) $100 \mu \mathrm{m}$ wide. Reprinted from reference [97], with permission from American Chemical Society, 2020.

As already mentioned, the presence of magnetic nanoparticles and dipole-dipole interactions lead to changes on both the rheological and hyperthermia properties. For instance, even without a magnetic field, the presence of colloids induces an increase of the fluid viscosity associated with the increased rate of energy dissipation during viscous flow $[37,79]$. The application of a magnetic field allows controlling the hydrodynamic flows and rheological properties of the magnetic fluids, as the hydrodynamic behaviour of the particles is changed. For example, in monodisperse ferrofluids of non-interacting short chains, the applied field parallel to the flow velocity gradient leads to a significant increase of both the effective viscosity and time of hydrodynamic relaxation [80]. In real polydisperse ferrofluids, the formation of heterostructures and their alignment with the magnetic field can lead to an enhanced magnetoviscosity effect compared to systems of individual particles $[82,98]$. However, above a certain flow velocity gradient, the aggregates break and the effective viscosity drops $[81,98]$. Furthermore, in ferrofluids under weak shear flows, the chain-like structures formed due to dipole-dipole interactions cause an increase of magnetization when subjected to a homogeneous magnetic field, while it decreases at strong shear flows [98]. Nonetheless, the magnetoviscous effect depends on various parameters, such as nanoparticle magnetic moment, size, size distribution and concentration (non-Newtonian behaviour is observed at moderate concentrations) [37].

\subsubsection{Implications of magnetic dipole-dipole interactions in magnetic hyperthermia}

Magnetic nanoparticles relaxation occurs through a Néel or Brownian mechanism, as referred to in previous sections. The Néel relaxation is strongly size-dependent, occurring at sizes that only require a little bit of energy to induce rotation of the magnetic moment, without changing the particle orientation:

$$
\tau_{N}=\tau_{0} e^{\frac{K V}{k_{B} T}}
$$

where $\boldsymbol{\tau}_{\mathbf{0}}$ is a characteristic time of the material $\left(10^{-9}-10^{-12} \mathrm{~s}\right), \boldsymbol{K}$ is the anisotropy constant, $\boldsymbol{V}$ the particle volume, $\mathbf{k}_{\mathbf{B}}$ the Boltzmann constant and $\mathbf{T}$ the temperature. The other mechanism is the Brownian relaxation, which consists of the physical rotation of the magnetic nanoparticle and is affected by the medium viscosity:

$$
\tau_{B}=\frac{3 \eta V_{H}}{k_{B} T}
$$

where $\boldsymbol{\eta}$ is the medium viscosity and $\mathbf{V}_{\mathbf{H}}$ the particle hydrodynamic volume. Upon application of an alternating magnetic field (AMF) to a medium containing non-interacting magnetic nanoparticles, which magnetization varies linearly with the applied magnetic field (low amplitude fields, where the response is linear in accordance with the linear response theory), heating occurs (magnetic hyperthermia) as a consequence of the delay of the magnetic nanoparticles' relaxation time compared to the oscillating AMF $[99,100]$. The dissipation power is proportional to the area of the hysteresis loop and is expressed according to the equation $[90,100]$ :

$$
\mathbf{P}=\mu_{0} \chi_{0} \pi \mathbf{f} \mathbf{H}^{2} \frac{\omega \tau}{1+(\omega \tau)^{2}}
$$

where $\mathbf{H}$ and $\mathbf{f}$ are the amplitude and frequency of the $\mathrm{AC}$ magnetic field, respectively, $\mu_{0}$ is the vacuum magnetic permeability, $\chi_{0}$ is the actual susceptibility and $\boldsymbol{\tau}$ is the effective relaxation time, which is dependent on both Néel and Brownian relaxations.

Magnetic hyperthermia consists on the exposure of the tissues to high temperatures $\left(42-45^{\circ} \mathrm{C}\right.$ ), either to kill tumor cells by apoptosis or to prompt higher susceptibility to radiation and antitumor drugs $[101,102]$. The thermal energy promotes denaturation of the cytoplasmic membrane and nuclear proteins required for the synthesis of DNA [101]. Thus, the cellular division can be hindered as a result of the higher susceptibility of cancer cells to heat [101].

A temperature above $42{ }^{\circ} \mathrm{C}$ stimulates the sensitivity to hyperthermia as a consequence of the reduction of blood flux, and the tumour tissue characteristics, such as the depleted oxygen and nutrients levels, low $\mathrm{pH}$, random vascularity and associated deficient heat dissipation $[32,101]$. On the other hand, a higher blood flux (temperature lower than $42{ }^{\circ} \mathrm{C}$ ) affords a synergy with chemotherapy as it increases oxygenation, drug accumulation, intracellular assimilation, and DNA damage. Therefore, a compromise around $42{ }^{\circ} \mathrm{C}$ is needed, and the combined use of hyperthermia and chemotherapy will synergistically enhance the antineoplastic drugs cytotoxic effect and perfusion facilitation [102].

The heating efficiency (specific loss power, SLP) is defined as the ratio of power dissipated per mass of the nanoparticle. However, 
the SLP is an inaccurate parameter for comparison of reported literature values, as it depends on $\boldsymbol{H}^{2}$ and frequency $(\boldsymbol{w})$ of the alternating magnetic field. Thus, a new parameter, the intrinsic loss power (ILP) was defined, which is the SLP normalized by the AC field strength and frequency [99].

However, when dealing with biological systems, the maximum $\boldsymbol{H} \boldsymbol{f}$ is limited to $\mathbf{5} \times \mathbf{1 0}^{\mathbf{9}} \mathbf{~} \mathbf{A} / \mathbf{m}$ in order to avert the undesired occurrence of eddy currents in biologic tissues. Thus, the heating power losses have to be maximized, not only considering this problem but also to reduce the required amount of nanoparticles to prevent any toxic side effect [103].

Further, it should also be pointed out that tissues have different thermal conductivity and thermal thresholds, which must be considered in future developments of magnetic hyperthermia [104106]. For example, Engelmann et al. [107] demonstrated that tissue damage depends not only on the bulk temperature and duration of treatment, but also on the cell type and thermal energy deposited per cell during treatment. Concerning the inconvenient thermal control through the dosage of nanoparticles, as large dosage can induce excessive temperature and low dosage might not be enough to kill cancer cells, Tang et al. [108] have recently demonstrated that a simple intermittent time-set technique could be used to control and maintain the enhanced temperature during the magnetic hyperthermia in cancer therapy.

The different parameters associated with the magnetic relaxation mechanisms leads to hyperthermia being affected by various structural aspects of nanoparticles, such as size, composition, shape, exchange-coupling, and the formation of assemblies (chains and rings) $[32,99,100]$. For instance, for a given frequency, there is an optimum size that displays maximum absorption of magnetic energy, i.e. the imaginary component of the magnetic susceptibility is maximized, which occurs when the characteristic fluctuation time is close to the period of the excitation field ( $\boldsymbol{w} \boldsymbol{\tau} \approx \mathbf{1}$ ). Yet, it has to be considered that for small sizes, magnetization is highly reduced due to the surface and internal spin canting effects (e.g. the formation of spin glass-like layers associated with the spin-spin exchange coupling) and the incomplete coordination of surface metal ions.

Despite the possibility of tuning nanoparticle' size, shape and composition, nanoparticles are prone to clustering, aggregation and self-assembly in biological systems, and also in magnetic gels. This aggregation, besides the inhomogeneous distribution in biological systems (e.g. accumulation in biological compartments) [109], will lead to changes in the magnetic behavior, and consequently the heating efficiency of both soft and hard magnetic nanoparticles [110-113]. Hereby, the magnetic dipole-dipole interactions need to be explored as a means to tune and control the heating efficiency.

Under high concentrations and an alternating magnetic field, nanoparticles can assemble in chain structures as a result of the dipolar coupling, which uniaxial anisotropy leads to higher hysteric losses, and thus an increase of heating efficiency at most angles between the field direction and cluster morphology anisotropy axis $[78,99]$. These structures display increased ferromagnetic behaviour with their magnetic moments locked in the direction of the magnetic field. Nonetheless, coalescence and cluster formation can lead to the reduction of heating efficiency. These clusters are characterized by randomly oriented anisotropy axis with regards to the direction of the external magnetic field that restricts the angle between the easy axis and magnetization vector. Such structure leads to a reduction of magnetic susceptibility and of the area of the hysteresis loop, and consequently a decrease of heating efficiency [99,114]. For instance, Niculaes et al. [115] developed different structures comprising oleic acid-coated iron oxide nanocubes: single nanocubes, dimers, trimers, and centrosymmetric structures (containing more than 4 nanocubes). The authors demonstrated that dimers and trimers attained higher SLP values than single nanoparticles and centrosymmetric nanoparticles. Such evidences the importance of dipolar interactions and cluster shape anisotropy in modulating the heating efficiency [76]. When dealing with isotropic clusters, improvement of heating efficiency can be attained with anisotropic particles or through the use of particles with higher saturation magnetization. Nonetheless, a decrease of heating efficiency for chain-assembled polydisperse nanoparticles has been reported [77]. Here, the authors evaluated some nanosystem design strategies, where the nanoparticles inside the magnetoliposomes under an applied field are represented by a longitudinal-type configuration, while in nanospheres are the random-type, as the particle rotation is inhibited. The authors reported a decrease in SLP in chains, which reaches a lower value in the random axes chain configuration, besides a drift of optimum hyperthermia to lower particle sizes.

Thus, considering the here discussed properties, a design of magnetic gels where non-interacting particle' rotation is not severely hindered or where nanoparticles are magnetically aligned in chain-to-tail configuration might offer a viable approach in the design of nanosystems. For more information on the effect of interparticle interaction in magnetic hyperthermia, the references [41], [99], [100], [116] and [117] are recommended.

\subsection{Hydrogels}

The field of hydrogels has been an area of intensive research in materials science since 1960, with the pioneering work of Wichterle and Lim [118]. The interest is highly associated with their similarity to biological tissues. For instance, soft tissues (e.g. skin, mucosa, brain, cartilage and muscle) are natural hydrogels [119]. Further, the high-water content, flexibility, biocompatibility and versatility have contributed to the use of hydrogels in a wide range of applications, such as tissue engineering [120-123], biosensors $[124,125]$, hygienic products [126], drug delivery [127,128], contact lenses $[129,130]$, cell therapy and 3D cell culture [131-134].

Hydrogels are elastic three-dimensional networks made of watersoluble polymers/small molecules $[131,135,136]$. The network can consist of natural polymers, that include anionic (e.g. carrageenan), cationic (e.g. chitosan), ampholytic (e.g. fibrin and collagen) or neutral (e.g. agarose) polymers [137]. Alternatively, the polymer can be synthetic, which include the commonly used polymers poly(vinyl alcohol), poly(ethylene glycol), poly( $N$-vinyl pyrrolidone)s, poly( $N$-isopropylacrylamide), poly(hydroxyalkyl methacrylate), poly(acrylamide), poly(acrylic acid) and poly(vinyl imidazole) [137]. Nonetheless, the polymer can also be a hybrid, such as alginatepolyethylene-glycol acrylate [138].

\subsubsection{Formation and properties of hydrogels}

The leading interactions involved in the network cross-linking or entanglement promote their water insolubility accompanied by the retention of a huge portion of solvent, that ultimately results in gel formation $[136,139]$. Hereby, the gelation process is characterized by a network expansion due to the capillary, osmotic and solvation forces that are counterbalanced by forces that resist expansion and keep network integrity [132]. The equilibrium between these opposing forces and its magnitude will determine the hydrogel 
intrinsic properties, such as the internal transport, diffusion characteristics and mechanical strength, which are also governed by the network morphology and chemical nature of the network chains.

The volume transition associated with gelation can be controlled by a wide variety of physical and chemical stimuli, such as temperature, $\mathrm{pH}$, sound, light, pressure, ionic force, electric or magnetic field [132]. Moreover, as the three-dimensional structure can be driven by physical or chemical interactions, the swelling and mechanical properties are differently affected according to the gelation mechanism. In chemical hydrogels, cross-linking occurs through covalent bonds, while, in physical hydrogels, is due to noncovalent interactions.

The deformability favors the adaptation of a hydrogel shape to a surface, while the muco- and bioadhesive properties strengthen its attachment, which can be further reinforced if cationic groups are present. These groups will also interact with DNA negative charges and can be explored on the development of non-viral vectors or bioinks for 3D cell culture and biofabrication [131,135,140,141].

Therefore, the main strategy is centered in taking advantage of the vast diversity of usable polymers, producible physical forms and stimuli capable of activating hydrogel formation and conceive the required physical and chemical properties [131,135,142,143]. This versatility is highly relevant in the development and tailoring of nanosystems, as it allows precise control over drug diffusion and the range of drugs capable of being incorporated through changes of the network structure porosity, the hydrogelator affinity to the aqueous environment and the density of reticulate agents $[131,144]$. These properties span the macroscopic scale characteristics of hydrogels, i.e. the size and porous structure, as they influence the administration route and macroscopic physical properties, such as the deformability [145]. At the mesh scale, the open spaces size (mesh size) affects the temporal and stimulusresponsive drug diffusion inside the hydrogel network, while at the molecular and atomistic scale, the network chains might have physical or chemical binding sites that interact with the loaded drug (covalent conjugation, electrostatic interaction or hydrophobic associations) and can affect the sustained or on-demand drug release.

Despite being less explored in magnetic gels, self-assembled (physical) hydrogels have acquired a remarkable plethora of applications, owing to their high portion of water and physicalchemical similarity to the cellular matrix, both in composition and mechanical properties, but also due to its cost-effective, versatile, facile, stable and robust structures [133,146-152]. Here, the gelation is achieved through the cooperative effect of different noncovalent intermolecular interactions, such as hydrogen bonding, van der Waals, electrostatic, hydrophobic and aromatic $\pi-\pi$ interactions [135]. A fundamental aspect from a physicochemical point of view is that the molecule must be amphiphilic, i.e. needs a hydrophilic region that interacts with water and a hydrophobic region that aggregates into a core [152]. A direct consequence of the wide variety of interactions involved in the gelation is that the design, preparation and manipulation of the hydrogelators must be rationally conceived, since it will be affected by several factors, such as $\mathrm{pH}$ value, kinetics of $\mathrm{pH}$ drop, temperature, ionic force and structure amphiphilicity [9-10,14,147-151]. Nevertheless, such aspects become an advantage, as the design can be rationalized from a library of compounds with the potentiality to be further modified with responsiveness to enzymatic, hydrolytic or environmental pathways. Particularly, supramolecular hydrogels are a class of physical hydrogels that comprise low-molecular weight hydrogelators such as oligopeptides, amphiphilic peptides and peptides conjugated to an aromatic moiety, or polymers that self-assemble into hierarchically-organized structures [153,154]. The reversible and spontaneous self-assembly of the initially randomly-oriented hydrogelators produces stable and well-defined structures due to localized inter- and intramolecular interactions, which will also affect the interaction of the resulting supramolecular architecture with other molecules, cells and tissues [154-156]. For instance, small peptides do not suffer from the structural complexity of therapeutic proteins [156], making it easier to stablish structure-function relations. Further, through modulation of the peptide blocks, it is possible to control the degradation and diffusion properties of hydrogels and even adapt it to encapsulate biological entities, such as enzymes.

\subsubsection{Application of hydrogels in drug delivery}

The controllable porous structure of hydrogels can be adapted to favor encapsulation of drugs in the matrix, besides modulating its release through changes that affect its diffusion coefficient, which guarantees a high drug concentration in the target site, for a long period of time [133]. Considering the abovementioned hydrogel properties, the applicability in different forms such as rectal [157], nasal [136], topic and intravenous injection can be optimized by adapting the mechanical and shape properties that best fit the required parameters to maximize the overall efficacy and patient compliance (Figure 5).

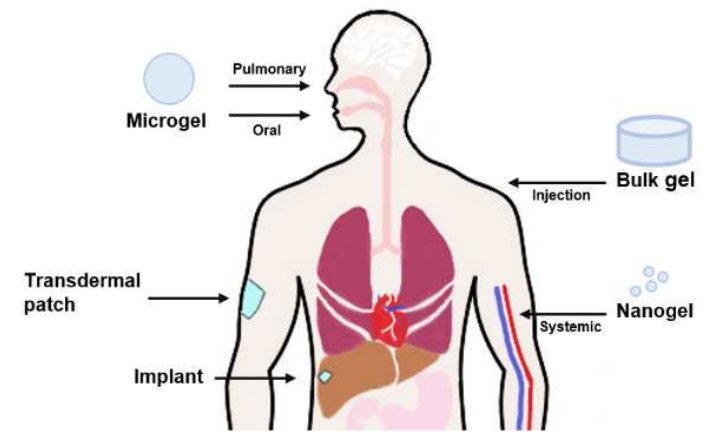

Figure 5. Schematic representation of the possible and optimum administration routes for each hydrogel shape

Macroscopic hydrogels are adequate for surgical implants, transepithelial and transdermal drug delivery, which is required when the target site is located deep within the tissue or when the biological barriers display low permeability to the drug to be administrated [145]. However, owing to the associated risks of surgical implantation, the alternative administration route of macroscopic hydrogels has been the in situ-gelation, shear-thinning hydrogels (flow when a shear stress is applied) or shape-memory hydrogels (can collapse and recover the initial structure). A minimal invasive solution is the use of microgels or nanogels, which, due to the small size, are needle-injectable, lead to facile natural clearance and enhance penetration through the physiological barriers, thus providing alternative routes such as oral, pulmonary, intrabony and systemic drug administration [145].

Currently, the development of hydrogels focuses on enhancing drug delivery efficiency by improving control over drug release rate and amplifying the range of compounds passible of being encapsulated [131,144]. The former is performed through modulation of the interaction between the hydrogel matrix and the drug of interest, while the latter requires the modification of the 
drug release diffusion barrier that is affected by the mesh size [131]. Release predominantly occurs through diffusion and is independent on the mesh size when the drug size is smaller than the mesh size, while steric hindrance becomes prominent for mesh sizes close to the drug size, which slows diffusion owing to frictional drag on the diffusing drug [155]. If the drug size is larger than the mesh size it becomes immobilized and release will occur either by mesh degradation, deformation or swelling [145].

Although hydrogels possess a hydrophilic matrix that might difficult the encapsulation of hydrophobic compounds, the hydrogel matrix can be tailored with hydrophobic domains [144]. Therefore, the chemical or physical stimuli responsiveness, reduced chemotherapeutic drug cytotoxicity and increased drug concentration on the therapeutic target, provides a highly advantageous and controllable nanosystem suitable for biomedical applications.

Further, the potential use of hydrogel-based materials for biomedical applications is demonstrated by the various formulations that have been translated to the clinical level and entered the market (examples in Table 1) [158-160].

Nonetheless, the development and characterization of new hydrogel formulations for clinical use must be previously accompanied by in vitro and in vivo studies. Several natural and synthetic polymers are now known to be non-toxic allowing to produce biocompatible hydrogels. Among the most common are chitosan [161], alginate [162], carrageenan [163], polyacrylamide [164], poly(vinyl alcohol) [165] and poly(vinyl pyrrolidone) [166]. The release of by-products from injectable biodegradable hydrogels or the presence of the biomaterial itself may cause toxicity or trigger an inflammatory response, respectively. Suggs et al. [167] tested a poly(propylene fumarate-co-ethylene glycol) hydrogel against endothelial cells and performed a cage implant system to assess the inflammatory response and reported that a higher content and molecular weight of PEG in copolymers lead to an increased cell viability and attenuated inflammatory reaction. Lopez-Silva and coworkers [168] demonstrated that multidomain peptide hydrogels with similar material properties, but different chemical functionalities, produce a different early inflammatory host response. The in vivo subcutaneous injection assay showed that negatively-charged peptides trigger a minimal inflammatory response characterized by low infiltration of immune cells and absence of vascularization and collagen deposition around and within the implant. The lysine-based hydrogel containing guanidinium ions promoted an acute inflammatory response that resolves over time and might be useful for tissue regeneration applications, while arginine-based hydrogels containing guanidinium ions induces a pro-inflammatory response that persists ten days after implantation.

For more information on hydrogels, the reader is referred to references [119], [169] and [170].

Table 1. Examples of hydrogels translated to clinical use, grouped by the type of hydrogel, drug and therapeutic application [158-160].

\begin{tabular}{|c|c|c|c|}
\hline Product & Hydrogel & Drug & Therapeutic application \\
\hline Vantas $^{\circledR}$ & $\begin{array}{l}\text { poly(2-hydroxyethyl methacrylate) and } \\
\text { poly(2-hydroxypropyl methacrylate) }\end{array}$ & Histrelin acetate & $\begin{array}{l}\text { Palliative treatment of prostate } \\
\text { cancer }\end{array}$ \\
\hline HYALO GYN ${ }^{\circledast}$ & $\begin{array}{l}\text { hyaluronic acid derivative, carbomer and } \\
\text { propylene glycol }\end{array}$ & - & $\begin{array}{l}\text { Vaginal dryness } \\
\text { Estrogen alternative }\end{array}$ \\
\hline TIMOPTIC-XE $^{\circledR}$ & Gellan gum derivative & Timolol malate & Glaucoma \\
\hline ELIGARD $^{\circledR}$ & poly(D,L-lactide-co-glycolide) & Leuprolide acetate & Prostate cancer \\
\hline REGRANEX & Carboxymethyl cellulose & Becaplermin & Diabetic foot ulcer \\
\hline Algisyl-LVR ${ }^{\circledR}$ & Alginate & - & Advanced heart failure \\
\hline JELMYTO ${ }^{\circledR}$ & $\begin{array}{l}\text { Hydroxypropyl methylcellulose, polaxamer and } \\
\text { polyethylene glycol }\end{array}$ & mitomycin & Upper tract urothelial cancer \\
\hline DEXTENZA $^{\circledR}$ & Polyethylene glycol & dexamethasone & $\begin{array}{l}\text { Ocular inflammation } \\
\text { Pain after ophthalmic surgery }\end{array}$ \\
\hline
\end{tabular}

\section{Development and properties of magnetic gels}

Magnetic gels can be described as materials that combine the magnetic and elastic properties of ferrofluids and hydrogels, respectively [171]. For instance, through an external magnetic field, the magnetic-elastic coupling allows the use of remote actuation [172]. Here, different actuation modes are possible, such as elongation, contraction, deflection, and coiling, which responses were observed to be induced within less than one second in magnetoactive polymeric gels, besides being proportional to the magnetic filler concentration [172-175]. Further, a magnetic field threshold (inversely proportional to filler concentration) was reported for the elongation and deflection responses, above which the response becomes non-linear and is characterized by a large deflection/elongation [172,173]. However, further increasing of the magnetic field strength only induces a small additional strain.

Concerning the fabrication of magnetic gels to attain the desired properties, several strategies have been developed. The main methods can be classified as blending method [176], grafting method [177], in situ precipitation [178], and swelling method [179]. These methods can be aggregated in polymer-first (in situ precipitation and swelling method) and particle-first (blending and grafting methods) strategies [35].

The blending method consists on a sequential preparation of the components, starting with the synthesis of the magnetic nanoparticles, which are then mixed with hydrogel precursors, and followed by polymerization [28]. Mikhnevich and coworkers [180] developed poly(acrylamide) ferrogels through the blending method. The authors used free radical polymerization of the monomer acrylamide and $N, N^{\prime}$-methylene-bis(acrylamide) as crosslinking agent under the presence of magnetic nickel nanoparticles. Despite the simplicity, the nanoparticles might interfere with the network formation and the final structure of the gel, besides the challenging uniform distribution of the nanoparticles and possible diffusion out 
of the gel upon swelling $[28,35]$. Thus, proper stabilization of the nanoparticles might be required.

The grafting methods (grafting-onto, grafting-from and graftingthrough) allow the development of magnetic gels crosslinked with nanoparticles [35]. Generally, these methods consist on the use of grafted/grafting magnetic nanoparticles with functional groups to form a covalent coupling with the monomers when polymerized [28]. Roeder and coworkers [181] developed magnetic gels of poly(acrylamide) with embedded methacrylate surfacefunctionalized spindle-like hematite nanoparticles (work as crosslinkers with the hydrogel matrix) through a grafting-through copolymerization (Figure 6A). The grafting methods have the advantage of coupling the magnetic nanoparticles to the hydrogel matrix, though the long, complicated and high-cost fabrication process restricts its use in the biomedical applications [182].

The in situ precipitation consists on the synthesis of magnetic nanoparticles inside the hydrogel network. Initially, after the gel is formed, it is placed into a concentrated aqueous solution of the required ions for the ferrite magnetic nanoparticles, until swelling equilibrium is reached, and then it is immersed into an alkali solution for precipitation of the magnetic nanoparticles [28]. Sang and coworkers [178] developed magnetic gels based on poly(2-acrylamido-2-methyl-1-propanesulfonic acid) (PAMPS) and iron oxide nanoparticles through immersion of the hydrogel in a concentrated aqueous solution of ferric and ferrous ions, followed by precipitation with an ammonia aqueous solution. However, this method is limited to hydrogels that possess stable networks, as it might be destroyed in the alkali solution.

In the swelling method, the prepared hydrogel is incubated with the ferrofluid, which is useful for the development of microgels.

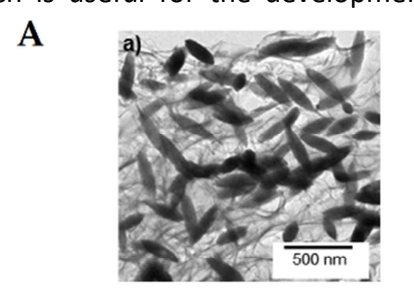

C I

B

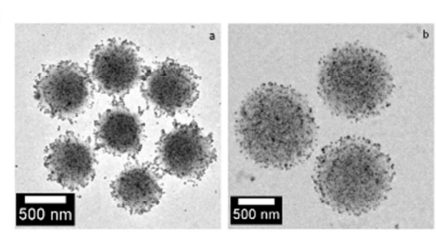

II
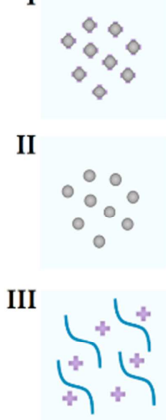

IV
Witt and coworkers [1179] developed poly( $N$-isopropylacrylamideco-allylamine $) \quad(\mathrm{P}(\mathrm{NIPAM}-\mathrm{co}-\mathrm{AA}))$ microgels comprising citrate-coated cobalt ferrite nanoparticles through the swelling method (Figure 6B), and evaluated the effect of two different microgel preparation strategies (batch and feeding method) on the homogeneity of the final magnetic microgel. The feeding method afforded homogeneously crosslinked microgels, while the batch method (which has a higher crosslinking density in the core) displayed an accumulation of magnetic nanoparticles in the outer shell. Nonetheless, not only the nanoparticles are limited to the hydrogel mesh size, but also need to be properly stabilized, as the interaction stablished with the network can affect the loading process. For more information on the fabrication of magnetic gels concerning biomedical applications, the references [27], [28], [35], [182] and [183] are recommended. A scheme of the discussed methods is displayed in Figure 6C. These methods are also common to the development of supramolecular magnetic gels $[24,69,184,185]$.

However, a current limitation in the development of supramolecular magnetogels compared to the polymeric is the long gelation time, which requires nanoparticles with high colloidal stability to ensure homogeneous gels. For instance, core/shell manganese ferrite/gold and gold-decorated nanoparticles afforded homogeneous dehydropeptide-based magnetogels of Npx-L-Met-Z$\triangle \mathrm{Phe}-\mathrm{OH}$ up to a concentration of $10 \mathrm{~m} / \mathrm{m} \%$ and $20 \mathrm{~m} / \mathrm{m} \%$ (mass of nanoparticle per mass of hydrogelator), respectively [24]. On the other hand, it was reported that stabilization of iron oxide nanoparticles with polyacrylic acid allowed homogeneous encapsulation of nanoparticles up to $30 \mathrm{~m} / \mathrm{m} \%$ in both hydrogels of

Npx-L-Asp-Z- $\Delta$ Phe-OH and Npx-L-Tyr-Z- $\triangle$ Phe-OH [69].
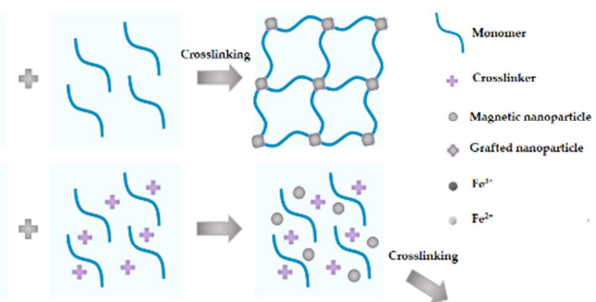

- Fe"

$\mathrm{Fe}^{2 \cdot}$
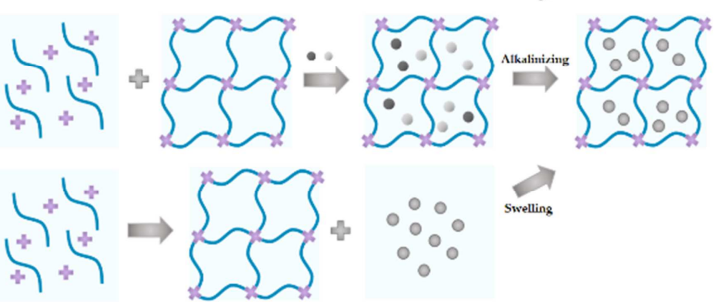

Figure 6. (A) TEM images of the magnetic hydrogels crosslinked with surface-functionalized spindle-like hematite nanoparticles. Adapted from reference [181] with permission from American Chemical Society, 2020. (B) TEM image of PNIPAM magnetic microgels, with the core microgel prepared from the batch method (left) and feeding method (right). Adapted from reference [179] with permission from American Chemical Society, 2020. (C) Schematic representation of the methods for preparation of magnetic gels: (I) grafting method; (II) blending method; (III) in situ precipitation method and (IV) swelling method.

Considering the discussed properties of the hydrogel component, magnetic gels can also be fabricated as magnetic nanogels [186,187], microgels [188-190], and macrogels [181,191]. Further, other modalities can be introduced, for instance, through the use of magnetic/plasmonic nanoparticles (plasmonic magnetogels) [24]. The interest of using plasmonic particles is associated with the phenomenon of localized surface plasmon resonance. This consists in charge density oscillations induced by an externally applied electric field that lead to an enhancement of local electromagnetic field around the particle, which can be used for sensing applications $[192,193]$, or photothermia due to the fast phase loss of the coherently excited electrons via electron-electron collisions [194].

As discussed by Weeber et al. [35], magnetic gels can be classified according with Kickelbick classification for hybrid materials [195], which are obtained through the discussed fabrication methods (Figure 7). Class I magnetic gels (Figure 7A and 
7B) include nanoparticles that weakly interact with the hydrogel network (also called blends) through physical interactions, such as nanoparticles embedded in the aqueous compartments or adsorbed onto the fibers. The class II magnetic gels (Figure 7C) include nanoparticles that strongly interact with the hydrogel fibers, such as covalent bonding or strong physical interactions. Another class is the nanoparticle containing micelles that self-assemble in ordered structures (micellar architecture) (Figure 7D) [196].

Concerning the requirement of understanding the behaviour of these materials to tailor with the desired properties, various theoretical studies have been carried out, including the use of molecular dynamics [197-206]. For instance, Lopez-Lopez et al. [207] evaluated the effect of the magnetic field-induced nanoparticle rearrangement on the shear elasticity of isotropic magnetic gels. The physical model predicts that rearrangement of interacting Néel particles (that do not turn around) will induce a decreasing dependence of the effective shear modulus if the magnetically induced anisotropy is insignificant, while for a strong anisotropy the modulus increases with the applied field. In another work, the presence of chain-like structures (anisotropic gels) was studied, which displayed an enhancement of the mechanical rigidity upon application of a magnetic field perpendicular to the sample shear [208]. These models are in agreement with experiments as for example, an enhancement of storage modulus under moderate magnetic fields was observed for alginate gels containing clusters of magnetic microparticles homogeneously distributed [209]. Further, simulations demonstrated larger elastic modulus enhancement for anisotropic gels than isotropic, besides being stiffer and displaying lower field-induced variation of mechanical module [210].
Particle chains assumed to be coated with a surface layer stiffer than the bulk matrix and strongly coupled to the matrix, were demonstrated to buckle into a wave-like shape as a means to reduce the magnetic energy upon the application of a magnetic field perpendicular to the chain orientation, which was concomitantly hindered by the matrix deformation (as it costs elastic energy) [211].

Different simulation approaches have been used to study the magnetic gels properties at different scales (from micro- to macroscopic) [212], such as full continuum-mechanical approaches [213], an elastic background continuum (particles are explicitly modelled) [214] or elastic spring matrix [215,216], an explicit approach [171], and density functional approach [200]. For instance, molecular dynamics has helped the understanding of magnetic field-induced deformation experimentally observed, which requires a large concentration of nanoparticles $[171,218,219]$. Weeber et al. studied the magnetic nanoparticlehydrogel coupling both in 2D [220] and 3D [90], through coarse-grained molecular simulations. In the $2 \mathrm{D}$ models, the crosslinked nanoparticles induced an isotropic deformation (shrinkage) under an external field, while in 3D models the deformation is anisotropic, as chains attached parallel to the rotation axis are not affected (Figure 7E and 7F). The authors observed a contraction parallel to the field, while an expansion occurred perpendicular to the magnetic field. This effect was associated with the high Poisson ratio (a measure of the contraction perpendicular to a strain that elongates the gel), which compensates the shrinkage that would otherwise occur due to the wrapping of the polymer chains around the particles.
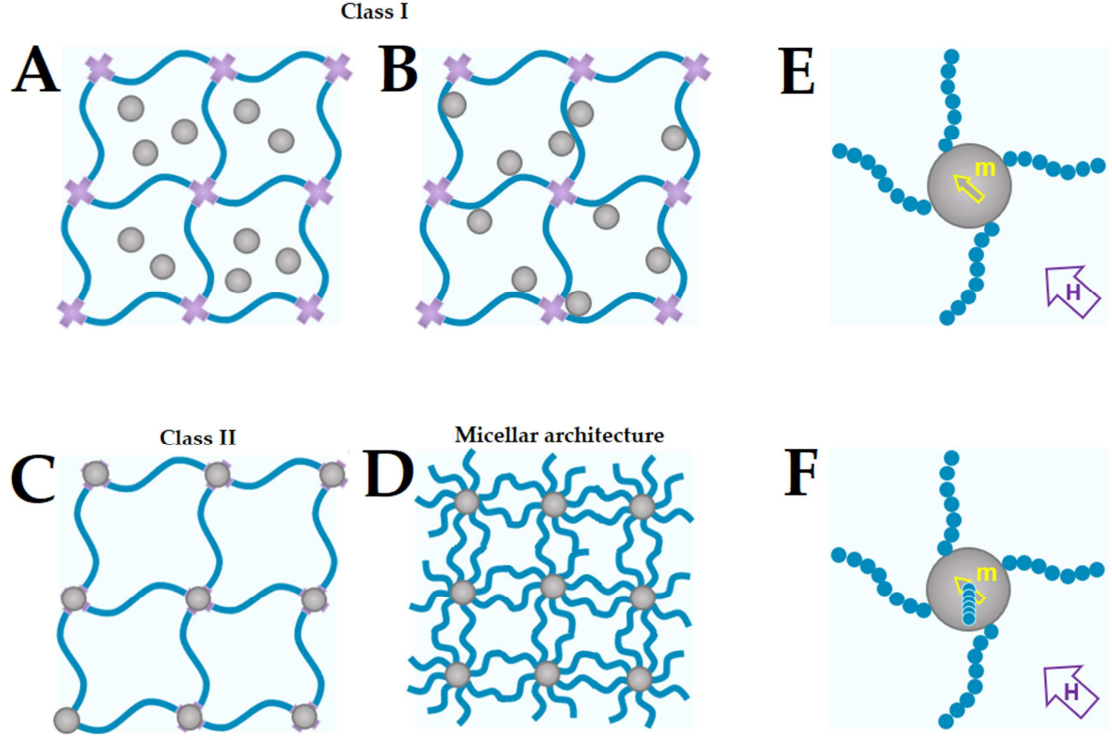

Figure 7. Schematic representation of magnetic gels (A and B) class I, (C) class II and (D) micellar architecture. Schematic representation of magnetic nanoparticles in a gel (E) 2D and (F) 3D model under the application of a magnetic field. In the 3D model, only the polymer chains in the plane of the magnetic field are affected.

The simulations studies also demonstrated that stronger dipoledipole interactions promote shrinkage of microgels, and that stiffness increases with the degree of crosslinking $[90,218]$, besides increasing the penalty on the alignment of the nanoparticles, which reduces the magnetic response comparatively to non-interacting magnetic particles. In microgels, a strong decrease of the magnetic susceptibility can be further associated with the rearrangement of nanoparticles chains in close-structures [218].

Macroscopic magnetic gels have been experimentally and theoretically demonstrated to display a hysteresis magnetization curve (while ferrofluids are superparamagnetic for small nanoparticles) [221,222]. Such was associated with the nanoparticles' rearrangement into chain-like aggregates, which size 
leads to the hysteretic dependence of the magnetization and susceptibility. A peak of susceptibility (as the applied field increases) is explained by the competition between the chaining, that reduces the demagnetization factor as the chain length increases, and the saturation of magnetization, that induces the decrease of the composite susceptibility [222].

This rearrangement of nanoparticles is also of special importance in gels of class I, where no direct coupling to polymer exists, i.e. the moments realign through Néel mechanism or only isotropic particle-polymer interactions are involved [89]. Here, the magnetic moments co-align and attract each other in the direction parallel to the magnetic field, while a repulsion occurs perpendicular to the field, leading to a deformation of the gel. For instance, the alignment of chain-like aggregates leads to an elongation of the gel parallel to the field and a contraction perpendicular to it.

Besides the microstructure and particle distribution, the deformation and response of the magnetic gels is also influenced by shape [223-227]. For example, ellipsoidal isotropic gels were demonstrated through simulations to elongate and align along the long axis to minimize the demagnetization energy, and a similar behaviour occurred for uniaxial gels with a microstructure aligned along the long axis [224]. If the particles chains are aligned along the short axis of a prolate, its alignment along the field (and elongation) showed to be favoured due to the favourable dipolar interactions (dipole-dipole and dipole-field) over the reduction of the demagnetization energy.

The discussed properties, including the magnetostriction of magnetic gels, have been of interest for various applications, such as the development of active scaffolds [228-230], magnetic hyperthermia [231,232], and drug delivery [233,234]. Savchak et al. [235] assessed the potential use of electromagnetic radiation close to the Ferro-Magnetic Resonance (FMR) frequency, which energy dissipation within the particles leads to heating of the gel and, consequently, a volume phase transition in thermoresponsive gels. The response depends on the particles concentration (variable as it is dynamic due to volume changes) and its chemical nature, and is regulated by changing the frequency of the electromagnetic signal, the time of exposure or the external bias field.

Besides the above discussed strategy, the use of DC and AC magnetic fields can also be used to control drug release. When a DC magnetic field is applied, the nanoparticles assemble in an end-toend configuration due to the attractive forces, which reduces the pore size and confines the drugs in the magnetic gel network, thus reducing its diffusion (figure 8A) [233]. In poly(vinyl alcohol) (PVA) magnetic gels, Lyu and coworkers [233] demonstrated that switching off the magnetic field resulted in a burst drug release due to reopening of the pores, and recovering of the normal drug diffusion after some time. Drug release induced by the AC magnetic field occurs through different mechanisms (see the magnetic hyperthermia - section 2.1.3.), where the generated heat (and consequent changes in structural or molecular configuration upon microstructural deformation of the hydrogel network) accelerates the drug release (figure 8C) [234].

$\mathrm{Hu}$ and coworkers [234] demonstrated that, upon exposure to an AC magnetic field, the chitosan-based magnetic gels displayed a burst drug release, which once switched off recovered the normal diffusion profile. In the absence of a magnetic field, the magnetic gels displayed a lower release rate than pure hydrogel, which suggested that nanoparticles can act as physical barriers to drug diffusion. Further, the authors reported that the amount of burst drug release progressively decreases after each $\mathrm{AC}$ cycle, due to a depletion of the loaded drug.

The hampered drug release in the absence of hyperthermia effect was also observed in the curcumin release from dehydropeptidebased plasmonic magnetogels (either containing core/shell manganese ferrite/gold or gold-decorated manganese ferrite nanoparticles) [24]. Similarly to the commonly used magnetic hyperthermia, the gels displayed an enhancement of drug release upon irradiation with wavelength above $600 \mathrm{~nm}$.
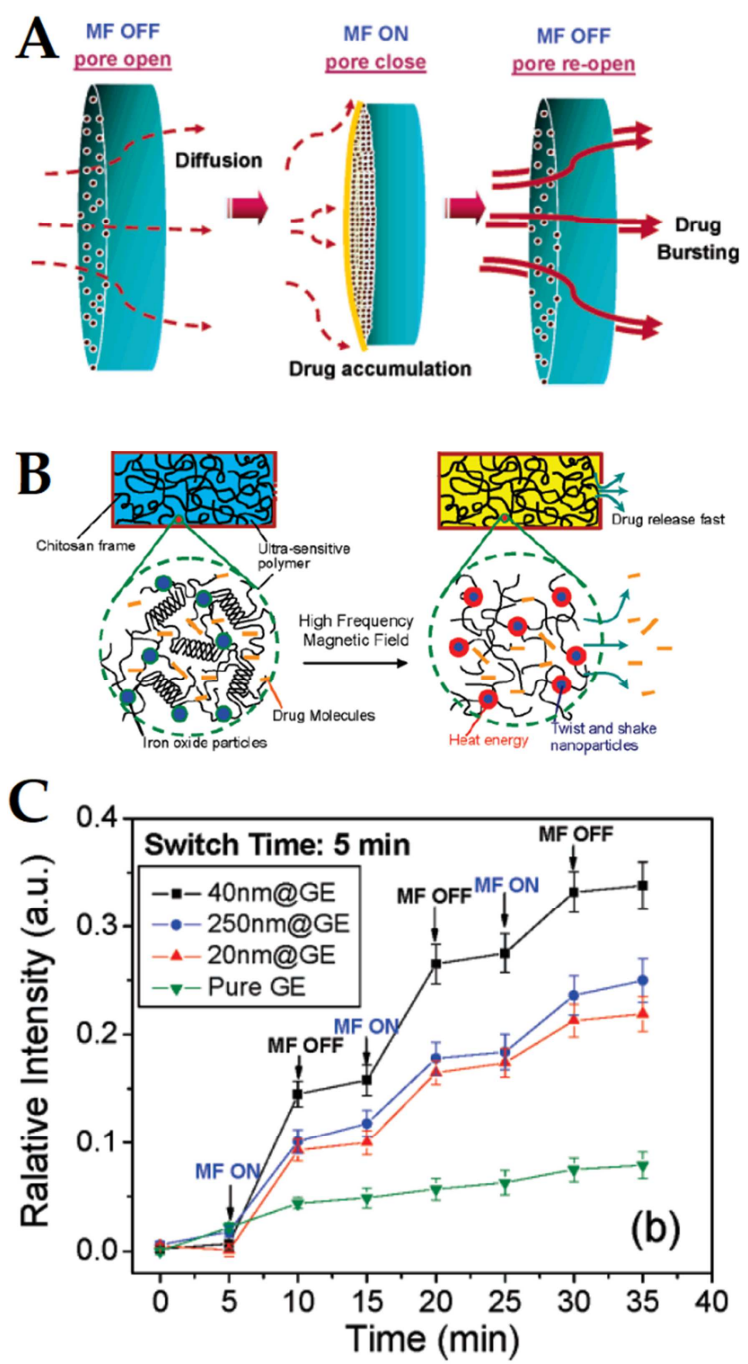

Figure 8. Schematic representation of the drug release mechanism through a (A) DC and (B) AC magnetic field. (C) Cumulative drug release profile of magnetic gels with $A C$ magnetic field exposure cycles. Figure $(A)$ was adapted from reference [233] with permission from American Chemical Society 2020 and figures (B) and (C) were adapted from reference [234] with permission from American Chemical Society, 2020.

Despite the hydrogel drawbacks, the magnetic gels improve some limitations and can also be fabricated to optimize some problems (also used in hydrogels), such as: the inhomogeneous loading of hydrophobic drugs is surpassed by the inclusion of hydrophobic domains, and the ensuring of its effective release can be attained through the magnetic response to a DC and AC magnetic field; the rapid drug release of hydrophilic drugs, which is a difficulty for gels with large pore sizes and high water content, can be solved with 
different physical and chemical interactions to enhance binding of the loaded drug; the existence of bulk gels, which are not easily deformed and have to be implanted, can be administered as micro and nanogels; and the need to avert unwanted drug release (e.g. during in situ hydrogels gelation period) upon administration through injection can also be solved through physical and chemical interactions with the magnetic gel components or use of composites. However, different complications might appear when dealing with all the described variables, and a possible solution that addresses all the discussed drawbacks is the combination with liposomes.

\section{Modulation of drug release through liposome- hydrogels}

As stated above, hydrogels are soft materials capable of mimic biological tissue, thus being extensively investigated in biomedical applications, such as tissue engineering [236-238], controlled drug delivery [239-241] and cancer therapy [242-245]. They are commonly referred as smart materials, as they can be composed of stimuli-responsive polymers, as well as incorporate magnetic nanoparticles (magnetic hydrogels) and therapeutic molecules. When using hydrogels as drug delivery systems, the diffusion pattern of molecules through the hydrogel matrix is an important factor to be considered upon the synthesis of the hydrogel, as it is influenced by the mesh size, the composition and crosslink density of the polymer network, and interactions that may occur between drugs and polymer chains [246, 247]. A fast drug release from a hydrogel is achieved if the drug molecules are smaller than the mesh size, while a slower diffusion of drugs occurs when the drug size approximates the mesh size. On the other hand, an ideal approach for deep tissue therapy is the immobilization of drugs on the polymer matrix of the hydrogel, which can be accomplished by designing hydrogels with pore sizes smaller than the drug size. Once it reaches the target tissue, drug release could be triggered by degradation, mechanical deformation or swelling of the polymer network [145]. For instance, the presence of magnetic nanoparticles in hydrogels allows the magnetic targeting of an injectable hydrogel and the remote-controlled release of drug molecules through mechanical deformation [248,249]. That is, the combination of superparamagnetic nanoparticles and a thermosensitive polymer allows the enhancement of drug release when an alternating magnetic field is applied, promoting a rise in temperature and the de-swelling of the hydrogel [250]. However, in this case, the de-swelling method can lead to a rapid burst release of drug molecules that might cause drug accumulation and toxicity $[30,251]$. One possible way to surpass the uncontrolled release of drugs, as well as liposomal instability in solution and loading of hydrophobic drugs, is the incorporation of liposomes into hydrogels. Several studies already concluded that drug-loaded liposomes included in hydrogels (liposomal gels) promote a prolonged and controlled drug release from the hydrogel due to a combined transport resistance of the liposome membrane and the polymer matrix [252-257]. The presence of liposomes can also increase the gel strength due to a higher bridging within the polymer matrix, which can lead to slower drug diffusion [253]. Large liposomes, such as large multilamellar liposomes with sizes higher than $280 \mathrm{~nm}$, can promote a decrease in the drug release kinetics and initial burst release, probably attributed to their immobilization in the hydrogel matrix or a slow diffusion coefficient [253]. On the other hand, liposome formulations with smaller sizes demonstrated a faster release that can result from a lower stability of the lipid bilayer. This means that the drug release rate can be tuned by modifying the liposomes size and composition [253]. This combination avoids the burst release effect and high concentration of drugs in plasma. Besides that, in vitro and in vivo studies that have been performed with liposomal hydrogels demonstrate their biocompatibility. Ren et al. [258] reported low cytotoxicity of doxorubicin-loaded liposomes (phosphatidylcholine and cholesterol) incorporated in chitosan/ $\beta$-glycerophosphate hydrogel. The same drug and liposomal composition were incorporated in a tri-block copolymer PLGA-PEG-PLGA to treat breast cancer locally [259]. The authors demonstrated in vivo biocompatibility and lower systemic toxicity of the drug within the hybrid system comparing with free drug. In addition, a hyaluronic acid hydrogel combined with celecoxib-loaded liposomes was used for intra-articular injection in rabbits with induced osteoarthritis. The insertion of liposomes allowed to improve pain control and cartilage protection [260].

In 2012, Lee and coworkers [252] developed hybrid hydrogels containing Doxorubicin-loaded liposomes embedded in a hydrophobically-modified chitosan network. The preparation consisted in the insertion of polymer hydrophobic moieties at the surface of small unilamellar vesicles, composed of dipalmitoylphosphatidylcholine (DPPC), that promote the gel formation through hydrophobic interactions, with the liposomes working as crosslinkers [261]. This hydrogel showed shear-thinning properties, which allow it to be used as injectable biomaterial, and promotes a slow and sustained release of drug over more than one week. Another interesting type of liposomal hydrogel assembly was presented by Kang et al. [262]. They prepared a hydrogel nanoparticle composed of hyaluronic acid modified with 1,2dimyristoyl-sn-glycero-3-phosphoethanolamine (DMPE) and methacrylic anhydride (MA), through an inverse microemulsion upon UV radiation, and with the purpose of creating a phospholipid assembly on the surface of the nanogel core, using lipid bilayers bearing amphiphiles (Figure 9).

Ciobanu et al. [255] prepared a nontoxic chitosan/gelatin hydrogel containing liposomes loaded with the hydrophilic dye calcein (multilamellar vesicles and small unilamellar vesicles). To achieve that, the drug-loaded liposomes suspension was added to the polymer solution before the addition of the cross-linkers (glutaraldehyde and sodium sulfate/sodium tripolyphosphate). In this study, the influence of important parameters on the drug release kinetics was studied. For instance, the release kinetics was slower in hydrogels containing sodium tripolyphosphate due to the increase of network density that leads to a slower diffusion of both multilamellar vesicles (MLVs) and small unilamellar vesicles (SUVs); calcein release efficiency is lower when the hydrogel has a higher concentration of chitosan (for both MLVs and SUVs) and hydrogels that incorporate MLVs release a higher amount of calcein, mostly due to their larger sizes allowing the storage of a higher concentration of drug. Also, it was concluded that the mechanism of drug release is performed by two steps: (1) slow diffusion of the liposomes and disruption of their lipid membrane releasing the drug and (2) diffusion of free drug towards the hydrogel matrix. 
A

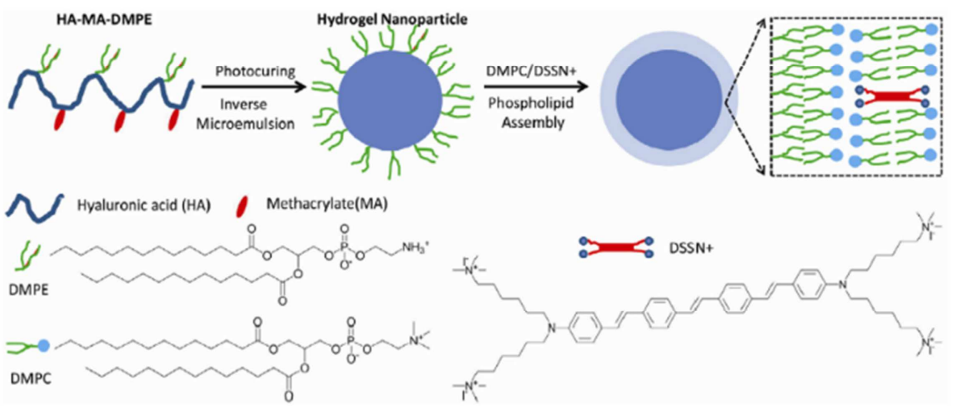

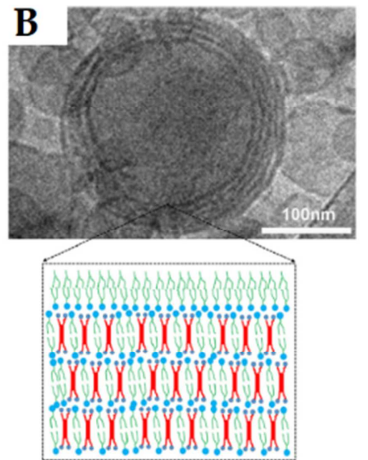

Figure 9. (A) Schematic representation of the assembling mechanism (inverse microemulsion) of a DMPC bilayer bearing DSSN+ conjugated oligoelectrolytes on the surface of hyaluronic acid nanoparticles modified with methacrylates and DMPE. (B) Cryogenic TEM image of the formed multilamellar vesicles composed of DMPC bearing DSSN+ on the surface of the nanogel core. Abbreviations: DMPE (1,2-dimyristoyl-sn-glycero-3-phosphoethanolamine); DMPC (1,2-dimyristoyl-sn-glycero-3-phosphocholine); DSSN+ (4,4'-bis[4'-(N,N-bis(6''-(N,N,N-trimethylammonium)hexyl)amino)styryl] stilbene tetraiodide). Reprinted from [262] with permission from Elsevier, 2020.

Drug release kinetics from liposomal hydrogels was also studied by Mourtas and coworkers [263]. In this study, the researchers compared the release of calcein (hydrophilic) and griseofulvin, a lipophilic drug, when included directly in hydrogels (control gels) or in liposomes embedded in the hydrogels (liposomal gels). Besides the drug lipophilicity, they tested the effect of three types of gel, (carbopol 974, hydroxyethylcellulose (HEC) and a mixture of both), and the lipid composition (phosphatidylcholine (PC) and distearoylglycero-PC/cholesterol (DSPC/chol)) on each drug release. The release rate of calcein from liposome is mainly affected by the rigidity of the lipid membrane and, thus, can be modulated using different lipid compositions. Here, the amount of both drugs released from both types of gel was higher in PC liposomes, due to their lower rigidity comparing with DSPC/chol liposomes. On the other hand, liposomal release of griseofulvin is not determined by the type of gel or lipid composition but by drug loading, being slower as the amount of drug loaded increases. This means that the $\log P$ value is also an important parameter to consider when the desired drug has amphiphilic or lipophilic properties. One year later, the effect of addition of liposomes in the rheological properties of the hydrogel was studied. The researchers found that a higher rigidity of the liposomal membrane modifies the flow and elastic properties of the gel (composed of Carbopol $974 \mathrm{NF}$ and Natrosol 250-HX) comparing with control gel (without liposomes), resulting in a higher viscosity that could influence drug release. Also, for rigid liposomes, in this case hydrogenated phosphatidylcholine, the zerorate viscosity and gel relaxation time increase with increasing liposome concentration and is independent of liposomes size [264].

A common approach to a selective and controlled drug release in lipogels (liposomal gels) is the use of triggering techniques. Lu et al. [265] described the combination of poly( $N$-isopropylacrylamide) (pNIPAM) microgel particle, a temperature-sensitive polymeric matrix, with lipid vesicles composed of 1,2-Dioleoyl-sn-glycero-3phosphocholine (DOPC), adsorbed at the surface of the spherical core of microgel. The triggered release strategy consisted in the response of pNIPAM to a rise in temperature above its lower critical solution temperature (LCST) of around $32{ }^{\circ} \mathrm{C}$. Transmission Electron Microscopy (TEM) and fluorescence microscopy images demonstrated the morphological transition of the lipogel to a smaller size, when temperature was increased from $22{ }^{\circ} \mathrm{C}$ to $37^{\circ} \mathrm{C}$.
This contraction is accompanied with a lipid release from the lipogel when the temperature is above the LCST (Figure 10A). The release kinetics of calcein incorporated in both native PNIPAM and lipogel systems showed that the presence of lipid assemblies allowed the retention of calcein release from ten minutes to two hours. Here, the hydrogel acts as drug carrier and the lipids as drug holders. Also, it was demonstrated that a salt-induced lipid release from the lipogel enables the modulation of the loading capacity and release of calcein, through the control of the lipid content.

Wang and coworkers [266] prepared a $\mathrm{pH}$-responsive hydrogel incorporating docetaxel (DTX)-loaded micelles, with the aim of producing an oral delivery system of this drug. For this, the micelles were based on amphiphilic poly( $\varepsilon$-caprolactone)- $b$-poly(ethylene glycol)- $b$-poly( $\varepsilon$-caprolactone) copolymer (PCEC) and the hydrogel was composed of co-monomers methoxyl poly(ethylene glycol)poly( $\varepsilon$-caprolactone)-acryloyl chloride (PECA), poly(ethylene glycol) methyl ether methacrylate (MPEGMA) and itaconic acid. The $\mathrm{pH}$ responsiveness was studied, and the hydrogel demonstrated an increasing swelling ratio as the $\mathrm{pH}$ changed from 2 to 8 , and a reversible swelling/deswelling behavior. The hydrogel showed a $\mathrm{pH}$-controlled diffusion of DTX-loaded micelles, being faster in simulated intestinal fluid than in simulated gastric fluid. This $\mathrm{pH}$-stimulated release allowed to increase the oral bioavailability up to $75.6 \%$. Also, this system was capable of inhibiting tumor growth in subcutaneous $4 \mathrm{~T} 1$ breast cancer model while decreasing systemic toxicity, when comparing with intravenous administration.

Recently, Ivashkov and coworkers [267] investigated the kinetics of a thermo-induced release of doxorubicin (DOX) encapsulated in anionic liposomes (1-palmitoyl-2-oleoyl-sn-glycero3-phospho-L-serine (PS)/phosphatidylcholine (PC)) complexed with a thermosensitive $\mathrm{N}$-isopropyl-acrylamide based cationic microgel. Here, the researchers synthesized microgels covered with liposomes with a liposome-to-microgel ratio range from 0.5 (unsaturated) to 30 (saturated). When the temperature increases from 25 to $50{ }^{\circ} \mathrm{C}$, the microgel collapses inducing DOX release. Two mechanisms for DOX release were proposed upon the thermoinduced collapse of the microgel (Figure 10B): (1) conformational changes of the polymer chains may occur leading to disruption of liposomes at the liposome-microgel interface (liposome-microgel interaction); (2) in saturated complexes, the decrease of the surface 
area promotes the squeezing of liposomes and subsequent disruption (liposome-liposome interaction). This means that, in saturated complexes, the release of drug is limited by the deswelling ratio of the microgel.

The importance of a rational development of liposomal gels carrying therapeutic agents is highlighted in the work of Faria et al. [268]. Here, the main goal was to produce a formulation for intravaginal co-delivery of tenofovir disoproxil fumarate (TDF) and

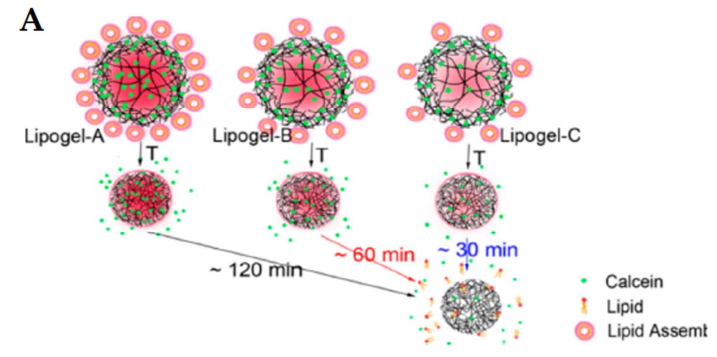

emtricitabine (FTC). Preliminary in silico and in vitro studies revealed that those drugs had distinct physicochemical characteristics that influence the choice of the nanocarrier. The proposed formulation consisted in TDF-loaded liposomes (1,2dioleoyl-sn-glycero-3-phosphocholine (DOPC), 1,2-dimyristoyl-snglycero-3- phosphocholine (DMPC), 1,2-dipalmitoyl-sn-glycero-3phosphocholine (DPPC) and DSPC) incorporated in a FTC-loaded carbomer-based hydrogel.

B

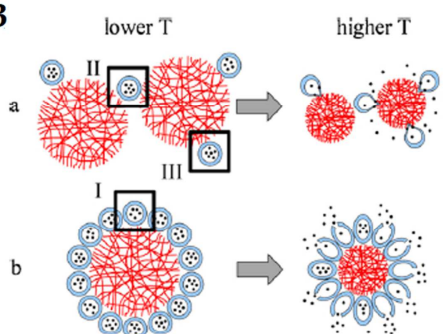

Figure 10. (A) Schematic representation of temperature-triggered release of calcein from lipogels with different lipid content at $37{ }^{\circ} \mathrm{C}$ (Lipogel-A, $\mathrm{B}$ and $\mathrm{C}$ ). The decrease in lipid content from $A$ to $C$ leads to a decrease in retention time of calcein in lipogels. Reprinted from [265] with permission from American Chemical Society, 2020. (B) Schematic representation of thermo-induced release of DOX from the lipogel via liposome-microgel interaction (a) or liposomeliposome interaction (b). Reprinted from [267] with permission from Elsevier, 2020.

More recently, Trusova and coworkers [269] assessed the drug release kinetics of DOX and europium coordination complex (V7) from an innovative type of hydrogel composed of neutral (phosphatidylcholine) and negatively charged (phosphatidylcholine and cardiolipin) multilamellar vesicles integrated in an amyloid network (egg-yolk lysozyme or bovine serum albumin fibrils (BSA)). They found that DOX encapsulation in the lipid vesicles is favored by the presence of BSA fibrils and its release from negatively charged vesicles was slower than that from neutral liposomes, meaning that electrostatic interactions have a high influence in the kinetics release of charged molecules. They also demonstrated that DOX release is governed by a non-Fickian diffusion that seems to relate to its location in the lipid polar/nonpolar interface of the liposomes.

Overall, liposomes seem to be a useful addition to hydrogels used in drug delivery, as they enable a longer retention of drugs in the composite avoiding undesirable secondary effects and decreasing cytotoxicity. The performance of these hybrid systems can be enhanced with a rational development and considering key factors like the ones described in this section.

\section{Magnetic liposome-hydrogels systems}

Magnetic liposomes surpass the difficulty of balancing a low passive permeability of the loaded cargo and a high release efficiency through a thermal trigger, as the magnetic nanoparticles enable an external triggered release and control over its dynamics [270]. Through magnetic hyperthermia, the membrane melting temperature $\left(T_{m}\right)$ can be reached, which induces a transition from the gel phase to the liquid crystalline phase that favours the permeability of the membrane to small compounds. Moreover, a low frequency $A M F$ can also be used to control drug release through membrane permeability changes induced by the nanoparticle motions in its vicinity [49].

Different architectures can be developed: (I) the nanoparticles contained in the inner aqueous cavity of the liposomes (aqueous magnetoliposomes); (II) the inner cavity is a cluster of magnetic nanoparticles (solid magnetoliposomes); (III) the nanoparticles are embedded into or onto the surface of the lipid bilayer [270-272]. Another strategy includes the development of coated magnetic nanoparticles, so that it works as multivalent ligands that cross-link vesicles into vesicle assemblies [273]. These assemblies can encompass different types of vesicles to deliver mutually incompatible or unstable bioactive molecules that mix upon release [273,274]. Haša et al. [274] developed assemblies comprising anionic liposomes, iron oxide nanoparticles and a positively charged polyelectrolyte (poly-L-lysine), which displayed responsiveness to an applied radiofrequency magnetic field. The authors demonstrated that the system could carry different payloads (resazurin and ascorbic acid, which are precursors of resorufin) independently, in separate liposomes combined at a defined stoichiometric ratio, which were released upon the magnetic stimulus, and in situ synthesis of resorufin occurred.

The combination of these systems with hydrogels into magnetic liposome-hydrogels (magnetic lipogels or magnetolipogels) can further improve the control over the materials' properties. For instance, the use of liposomes as reservoirs of the payload provides a reversible means for stimuli-responsive release [275]. However, the gelation conditions and the liposomes stability must be assessed for each formulation as the physical conditions and the nature of the materials can differ substantially. For example, liposomes can be stressed and destabilized during ionic gelation of alginate, which causes their leakage. Nonetheless, magnetic vesicle assemblies were successfully incorporated in alginate gels by Mart et al. [276], which not only respond to an AMF that enables the control over the release of the stored drugs or bio(macro)molecules, but can also be patterned by an external magnetic field, and host membrane-bound enzymes/glycolipids, thus leading to potential applications as smart scaffolds for stem cells or controlled drug delivery. Later, de Cogan et al. [277] demonstrated that this system could be used as a smart cell culture scaffold responsive to magnetic impulses. The system included 
dipalmitoylphosphatidylcholine (DPPC) vesicles assemblies loaded with ascorbic acid-2-phosphate (AAP), which is required to induce the production of extracellular collagen by chondrocytes and osteoblasts. The authors verified that upon the AMF induced release of $A A P\left(T_{m} \approx 42{ }^{\circ} \mathrm{C}\right.$ for DPPC), the production of collagen was switched on, which resulted in the addition of this extracellular matrix protein to the alginate scaffold (Figure 11A).

Besides the temporal control on the translation of magnetic signals into chemical information, the magnetic lipogels also display spatial control through the magnetic positioning. This was demonstrated by de Cogan et al. [278] that developed alginate magnetic lipogels based on vesicle assemblies loaded with nickel (II) that could support the growth of fibroblasts if fibronectin was present in the gels. Upon AMF application, nickel (II) diffused out of the vesicles and interacted with the alginate gel matrix, which slowed nickel (II) diffusion out of the hydrogel block. Through exploration of the slowed diffusion in the alginate gel and the magnetic patterning of the vesicles, the authors were able to attain spatial control over the apoptosis inside the gel. Despite the preferential use of alginate, magnetic lipogel based on chitosan and prepared through the blending method have also been reported [279].
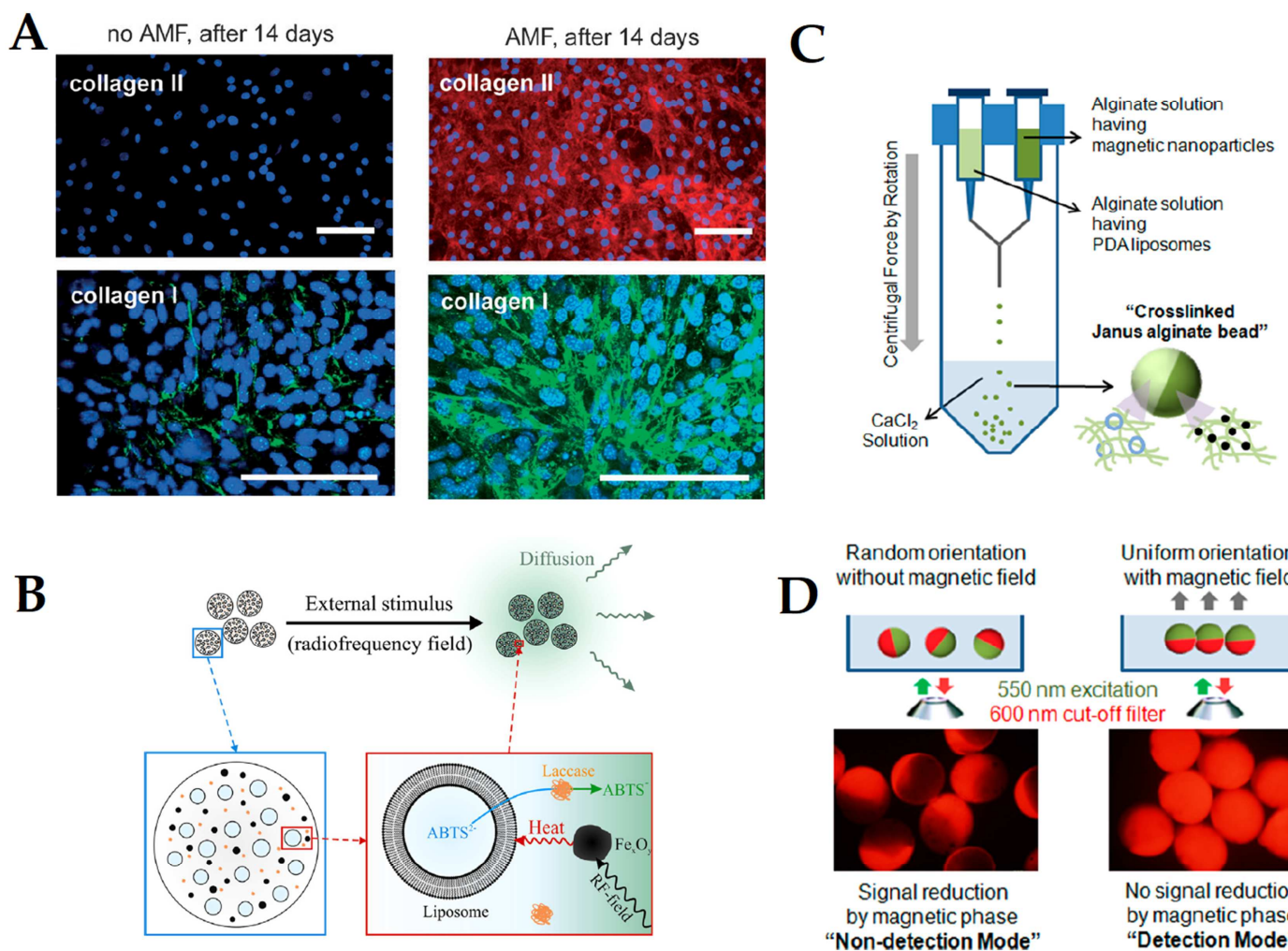

Figure 11. (A) Confocal microscopy images of the magnetic lipogels containing chondrocytes without (left) and with (right) an applied AMF. The cell nuclei are stained with fluorescent blue staining, collagen II is stained red with tetramethyl rhodamine isothiocyanate (TRITC)-secondary antibody, and collagen I is stained green with FITC-secondary antibody. Adapted from reference [277] with permission from Wiley Interscience, 2020. (B) Schematic representation of the RF-field triggered release of ABTS and consequent oxidation by laccase. Adapted from reference [283] with permission from Elsevier, 2020. (C) Schematic representation of the fabrication procedure of Janus particles containing PDA liposomes and magnetic nanoparticles. (D) Orientation of Janus microparticles under an externally applied magnetic field. Adapted from reference [284] with permission from American Chemical Society, 2020.

Similar to hydrogels and magnetic gels, the magnetic lipogels can also be developed as microgels, where the liposomes and the nanoparticles can be independently adjusted [280,281]. Hanuš et al. [280] developed alginate magnetic lipogel microparticles (40$80 \mu \mathrm{m})$ containing DPPC/Chol (2:1) liposomes through a drop-ondemand inkjet method, in which the surface can be further functionalized with a mesoporous silica layer, as suggested by the authors. In a work by Pittermannová et al. [281], alginate magnetic lipogel microparticles comprising DPPC liposomes were developed through an extractive gelation process implemented in a microfluidic device, which resulted in 5-10 $\mu \mathrm{m}$ microparticles. The authors also demonstrated that the system displayed temperature responsiveness as release only occurred when the particles were exposed to $45{ }^{\circ} \mathrm{C}$, thus providing an on-demand drug release mechanism.
The magnetic lipogels could also be used as contrast agents and provide real-time monitorization of the therapy. Elk et al. [282] developed alginate gels cross-linked with a $\boldsymbol{T}_{\mathbf{2}}^{*}$ magnetic resonance imaging (MRI) contrast agent (holmium ions) embedded with thermosensitive liposomes loaded with $\boldsymbol{T}_{\mathbf{1}} \mathrm{MRI}$ contrast agent $\left[\mathrm{Gd}(\mathrm{HPDO} 3 \mathrm{~A})\left(\mathrm{H}_{2} \mathrm{O}\right)\right]$ (ProHance $\left.{ }^{\circledR}\right)$. The authors reported that upon hyperthermia, a rapid release of the content occurs, which induce a higher peak concentration of the drug in the tumour. Here, the holmium ions, which act as cross-linkers of the alginate gel network, allow the visualization of the microgels by MRI, while the release of the loaded contrast agent in the liposomes can be explored as an indicator of the in vivo release of a cytostatic drug. A major advantage of the magnetic lipogels is the possibility of being used as bioreactors with spatiotemporal control. Ullrich et al. [283] developed alginate magnetic lipogels loaded with laccase in the hydrogel matrix and the substrate 2,2'-azinobis(3- 
ethylbenzthiazoline-6-sulfonate) (ABTS) in the liposomes (Figure 11B). The system displays (I) structural function provided by the alginate gel that immobilizes the components; (II) catalytic function due to the immobilized enzymes; (III) storage function, provided by the liposomes that act as reservoirs of the molecular cargo; (IV) and communication function through translation of radiofrequency signal into a biochemical reaction, due to the temporary higher permeability of the liposomes membranes.

Despite being less explored, Janus magnetic lipogel particles have also been reported in reference [284]. The authors fabricated alginate microbeads through a double syringe method (Figure $11 \mathrm{C}$ and 11D), comprising a compartment loaded with magnetic nanoparticles, and the other with 1,2-dipalmitoyl-sn-glycero-3galloyl (DPGG) and polydiacetylene (PDA) for the detection of lead (II).

Overall, the localized heating, large penetration depth and specificity of the radiofrequency (RF) field make these systems highly promising comparatively to other nanomaterials and stimuli strategies (e.g. light or $\mathrm{pH}$ triggered release). Further, the possibility of stoichiometrically compartmentalizing different precursors of biologically active compounds, that are unstable or highly reactive, makes these systems adequate for drug delivery, as they provide an opportunity to attain high therapeutic efficiency and efficacy without affording enough time for a pathogen to develop resistance [283].

\section{Conclusions and future perspectives}

The combination of hydrogels and magnetic nanoparticles into a single system, the magnetic gels, has led to a promising smart multifunctional material for biomedical applications.

Several experimental and theoretical studies have been carried out to understand the properties of these materials, and how they can be modulated and optimized for a particular application (e.g. shape, size, nanoparticle distribution, and nanoparticle-matrix interaction). Further combination with liposomes into magnetic liposome-gels (magnetic lipogels or magnetolipogels) provided structural, catalytic, storage and communication functions with spatiotemporal control, and allows real-time monitorization through MRI. Such properties make the magnetic lipogels ideal for different applications such as theranostic systems, controlled drug delivery of unstable molecules, as bioreactors and smart cell culture scaffolds.

Particularly, different limitations associated with the separate use of the components are surpassed in the magnetic lipogels. For instance, the burst drug release is avoided, providing a prolonged and controlled drug release that can be triggered and tuned through application of an alternating magnetic field. Considering that release can occur passively through diffusion of liposomes and posterior membrane disruption, or drug diffusion towards the hydrogel matrix, the drug release can be tuned by varying the membrane rigidity (e.g. lipid composition), and the hydrogel structure, such as the network and/or cross-linking density. However, a major limitation is the need to assess different liposome formulations for a specific hydrogel, as the matrix can induce the leakage of liposomes during gelation through destabilization of the lipid membrane. Nevertheless, the reported works have already advanced various advantages of magnetic lipogels, such as the stoichiometric loading of different compartmentalized payloads
[274], and spatial control over release from the hydrogel matrix [278], besides the fabrication of micro- and Janus particles [281,284].

Yet, besides a lack of studies on the effect of the composites in the rheological properties and the influence of different compositions in drug release, the majority of the reported works comprise polymeric hydrogels, whereas works on supramolecular magnetic lipogels are still lacking, which the variety of non-covalent interactions might lead to a complex behaviour. Other system architecture to be designed would comprise the magnetic gel embedded in the inner cavity of a liposome, which could be fabricated using microfluidics and a magnetic field to modulate the nanoparticle orientation, and consequently, the dipole-dipole interactions and the heating efficiency. From the magnetic perspective, other materials could be explored, such as the combination of magnetic and plasmonic particles to enhance the hyperthermia effect while using lower magnetic field and laser intensities [285], besides providing a means for the monitorization of drug release [286], and use of a new imaging technique, the magnetomotive photoacoustic imaging [287]. The magnetoelectric nanoparticles are also of interest owing to the control of the nanoparticle dipole moment and magnetic moment through a local magnetic field and electric field, respectively [288].

Overall, the discussed properties and the stoichiometric control over different reservoirs in magnetic lipogels can be helpful in the development of multimodal therapeutic strategies, which can comprise liposomes with different triggers or melting temperatures, enabling a sequential in situ administration of different therapeutic and/or diagnostic agents. Thus, the magnetic lipogels offer the opportunity to develop theranostic strategies that can potentially translate to clinical studies, besides the possibility of being used in other biomedical applications.

\section{Conflicts of interest}

There are no conflicts of interest to declare.

\section{Acknowledgements}

This work was supported by the Portuguese Foundation for Science and Technology (FCT) in the framework of the Strategic Funding of CF-UM-UP (UIDB/04650/2020). S. R. S. Veloso acknowledges FCT for a PhD grant (SFRH/BD/144017/2019). Support from MAP-Fis Doctoral Programme is also acknowledged.

\section{References}

1. M. Mohammadi, A. Nojoomi, M. Mozafari, A. Dubnika, M. Inayathullah, J. Rajadas, Nanomaterials engineering for drug delivery: a hybridization approach, J. Mater. Chem. B 5 (2017) 3995-4018. doi: 10.1039/C6TB03247H.

2. M. Wei, S. Li, W. Le, Nanomaterials modulate stem cell differentiation: biological interaction and underlying mechanisms, J. Nanobiotechnol. 15 (2017) 1-13. doi: 10.1186/s12951-017-0310-5.

3. B. Smith, S. Gambhir, Nanomaterials for in vivo imaging, Chem. Rev. 117 (2017) 901-986. doi: 10.1021/acs.chemrev.6b00073.

4. K. Bae, H. Chung, T. Park, Nanomaterials for cancer therapy and imagining, Mol. Cells, 31 (2011) 295-302. doi: 10.1007/s10059-011-0051-5. 
5. P. Ilg, Stimuli-responsive hydrogels cross-linked by magnetic nanoparticles, Soft Matter 9 (2013) 34653468. doi:10.1039/c3sm27809c.

6. S. Veloso, P. Ferreira, J. Martins, P. Coutinho, E. Castanheira, 2018. Magnetogels: Prospects and main challenges in biomedical applications. Pharmaceutics. 10, 145. doi:10.3390/pharmaceutics10030145.

7. M. Abrougui, M. Lopez-Lopez, J. Duran, 2019. Mechanical properties of magnetic gels containing rodlike composite particles. Philos. Trans. R. Soc. A. 377 , 20180218. doi:10.1098/rsta.2018.0218.

8. V. Molchanov, V. Pletneva, I. Klepikov, I. Razumovskaya, O. Philippova, Soft magnetic nanocomposites based on adaptive matrix of wormlike surfactant micelles, RSC $\begin{array}{llll}\text { Advances. } & 8 & \text { (2018) }\end{array}$ doi:10.1039/c8ra01014e.

9. R. Contreras-Montoya, A. Bonhome-Espinosa, A. Orte, D. Miguel, J. Delgado-López, J. Duran, J. Cuerva, M. Lopez-Lopez, L. Cienfuegos, Iron nanoparticles-based supramolecular hydrogels to originate anisotropic hybrid materials with enhanced mechanical strength, Mater. Chem. Front. 2 (2018) 686-699. doi:10.1039/c7qm00573c.

10. S. Bhattacharya, S. Samanta, Soft-nanocomposites of nanoparticles and nanocarbons with supramolecular and polymer gels and their applications, Chem. Rev. 116 (2016)

11967-12028. doi:10.1021/acs.chemrev.6b00221.

11. O. Ozay, S. Ekici, Y. Baran, N. Aktas, N. Sahiner, Removal of toxic metal ions with magnetic hydrogels, Water Res. 43 (2009) 4403-4411. doi:10.1016/j.watres.2009.06.058.

12. H. Hamidian, T. Tavakoli, Preparation of a new $\mathrm{Fe}_{3} \mathrm{O}_{4} /$ starch-g-polyester nanocomposite hydrogel and a study on swelling and drug delivery properties, Carbohydr. Polym. $144 \quad$ (2016) 140-148. doi:10.1016/j.carbpol.2016.02.048.

13. G. Mahdavinia, A. Mosallanezhad, M. Soleymani, M. Sabzi, Magnetic- and $\mathrm{pH}$-responsive $K$ carrageenan/chitosan complexes for controlled release of methotrexate anticancer drug, Int. J. Biol. Macromol. 97 (2017) 209-217. doi:10.1016/j.ijbiomac.2017.01.012.

14. A. Bonhome-Espinosa, F. Campos, I. Rodriguez, V. Carriel, J. Marins, A. Zubarev, J. Duran, M. Lopez-Lopez, Effect of particle concentration on the microstructural and macromechanical properties of biocompatible magnetic hydrogels, Soft Matter. 13 (2017) 2928-2941. doi:10.1039/c7sm00388a.

15. H. Kim, H. Park, J. Lee, K. Lee, Magnetic field-responsive release of transforming growth factor beta 1 from heparin-modified alginate ferrogels, Carbohydr. Polym. 151 (2016) 467-473. doi:10.1016/j.carbpol.2016.05.090.

16. Y. Kim, T. Kim, Y. Kim, D. Harbottle, J. Lee, Highly effective $\mathrm{Cs}^{+}$removal by turbidity-free potassium copper hexacyanoferrate-immobilized magnetic hydrogels, J. Hazard. Mater. $340 \quad$ (2017) 130-139. doi:10.1016/j.jhazmat.2017.06.066.

17. A. Ahmed, C. Zhang, J. Guo, Y. Hu, X. Jiang, Fabrication and characterization of Gd-DTPA-loaded chitosanpoly(acrylic acid) nanoparticles for magnetic resonance imaging, Macromol. Biosci. 15 (2015) 1105-1114. doi:10.1002/mabi.201500034.

18. T. Mitsumata, Y. Horikoshi, K. Negami, High-power actuators made of two-phase magnetic gels, Jpn J. Appl. Phys. 47 (2008) 7257-7261. doi:10.1143/jjap.47.7257.

19. S. Song, Y. Liu, A. Song, Z. Zhao, H. Lu, J. Hao, Peroxidase mimetic activity of $\mathrm{Fe}_{3} \mathrm{O}_{4}$ nanoparticle prepared based on magnetic hydrogels for hydrogen peroxide and glucose detection, J. Colloid Interface Sci. 506 (2017) 4657. doi:10.1016/j.jcis.2017.07.029.
20. J. Sang, R. Wu, P. Guo, J. Du, S. Xu, J. Wang, 2015. Affinity-tuned peroxidase-like activity of hydrogelsupported $\mathrm{Fe}_{3} \mathrm{O}_{4}$ nanozyme through alteration of crosslinking concentration. J. Appl. Polym. Sci. 133, 43065. doi:10.1002/app.43065.

21. J. Zhang, T. Yao, C. Guan, N. Zhang, X. Huang, T. Cui, J. $\mathrm{Wu}, \mathrm{X}$. Zhang, One-step preparation of magnetic recyclable quinary graphene hydrogels with high catalytic activity, J. Colloid Interface Sci. 491 (2017) 7279. doi:10.1016/j.jcis.2016.12.019.

22. N. Satarkar, W. Zhang, R. Eitel, J. Hilt, 2009. Magnetic hydrogel nanocomposites as remote controlled microfluidic valves. Lab Chip. 9, 1773. doi:10.1039/b822694f.

23. J. Kim, C. Chun, B. Kim, J. Hong, J. Cho, S. Lee, S. Song, Thermosensitive/magnetic poly(organophosphazene) hydrogel as a long-term magnetic resonance contrast platform, Biomaterials. $33 \quad$ (2012) 218-224. doi:10.1016/j.biomaterials.2011.09.033.

24. S. Veloso, J. Martins, L. Hilliou, C. O. Amorim, V. Amaral, B. Almeida, P. Jervis, R. Moreira, D. Pereira, P. Coutinho, P. Ferreira, E. Castanheira, Dehydropeptide-based plasmonic magnetogels: a supramolecular composite nanosystem for multimodal cancer therapy, J. Mater. Chem. B. 8 (2020) 45-64. doi:10.1039/c9tb01900f.

25. Z. Zhang, S. Song, Multiple hyperthermia-mediated release of TRAIL/SPION nanocomplex from thermosensitive polymeric hydrogels for combination cancer therapy, Biomaterials. 132 (2017) 16-27. doi:10.1016/j.biomaterials.2017.03.049.

26. H. Kim, A. Jo, S. Baek, D. Lim, S. Park, S. Cho, J. Chung, J. Yoon, Synergistically enhanced selective intracellular uptake of anticancer drug carrier comprising folic acidconjugated hydrogels containing magnetite nanoparticles, Sci. Rep. 7 (2017). doi:10.1038/srep41090.

27. N. Jalili, M. Muscarello, A. Gaharwar, Nanoengineered thermoresponsive magnetic hydrogels for biomedical applications, Bioengineering \& Transla. Med. 1 (2016) 297-305. doi:10.1002/btm2.10034.

28. Y. Li, G. Huang, X. Zhang, B. Li, Y. Chen, T. Lu, T. Lu, F. Xu, Magnetic hydrogels and their potential biomedical applications, Adv. Funct. Mater. 23 (2012) 660-672. doi:10.1002/adfm.201201708.

29. S. Hua, M. de Matos, J. Metselaar, G. Storm, 2018. Current trends and challenges in the clinical translation of nanoparticulate nanomedicines: Pathways for translational development and commercialization, $\begin{array}{llll}\text { Front. } & \text { Pharmacol. } & \text { 9, }\end{array}$ doi:10.3389/fphar.2018.00790.

30. S. Grijalvo, J. Mayr, R. Eritja, D. Díaz, Biodegradable liposome-encapsulated hydrogels for biomedical applications: a marriage of convenience, Biomater. Sci. 4 (2016) 555-574. doi:10.1039/c5bm00481k.

31. A. Akbarzadeh, M. Samiei, S. Davaran, 2012. Magnetic nanoparticles: preparation, physical properties, and applications in biomedicine, Nanoscale Res. Lett. 7, 144. doi:10.1186/1556-276x-7-144.

32. B. Issa, I. Obaidat, B. Albiss, Y. Haik, Magnetic nanoparticles: Surface effects and properties related to biomedicine applications, Int. J. Mol. Sci. 14 (2013) 21266-21305. doi:10.3390/ijms141121266.

33. G. Papaefthymiou, Nanoparticle magnetism, Nano $\begin{array}{llll}\text { Today. } & 4 & \text { (2009) } & \text { 438-447. }\end{array}$ doi:10.1016/j.nantod.2009.08.006

34. L. Kafrouni, O. Savadogo, Recent progress on magnetic nanoparticles for magnetic hyperthermia, Prog. biomater. 5 (2016) 147-160. doi:10.1007/s40204-0160054-6. 
35. R. Weeber, M. Hermes, A. Schmidt, C. Holm, 2018 Polymer architecture of magnetic gels: a review, J. Phys. Condens. Matter. 30, 063002. doi:10.1088/1361$648 x /$ aaa344.

36. T. Indira, P. Lakshmi, Magnetic nanoparticles - A review, Int. J. Pharm. Sci. Nanotechnol. 3 (2010) 1035-1042.

37. Schmidt, A. (2007). Thermoresponsive magnetic colloids, Colloid Polym. Sci., 285, pp. 953-966.

38. M. Bruschi, L. de Toledo, 2019. Pharmaceutical applications of iron-oxide magnetic nanoparticles, Magnetochemistry. doi:10.3390/magnetochemistry5030050.

39. W. Xie, Z. Guo, F. Gao, Q. Gao, D. Wang, B. Liaw et al., Shape-, size- and structure-controlled synthesis and biocompatibility of iron oxide nanoparticles for magnetic theranostics, Theranostics. 8 (2018) 32843307. doi:10.7150/thno.25220.

40. R. Andrade, S. Veloso, E. Castanheira, 2020. Shape anisotropic iron oxide-based magnetic nanoparticles: synthesis and biomedical applications, Int. J. Mol. Sci. 21, 2455. doi:10.3390/ijms21072455.

41. D. Lisjak, A. Mertelj, Anisotropic magnetic nanoparticles: A review of their properties, syntheses and potential applications, Prog. Mater. Sci. 95 (2018) 286-328. doi:10.1016/j.pmatsci.2018.03.003.

42. S. Mortazavi-Derazkola, M. Salavati-Niasari, O. Amiri, A. Abbasi, Fabrication and characterization of $\mathrm{Fe}_{3} \mathrm{O}_{4} @ \mathrm{SiO}_{2} @ \mathrm{TiO}_{2} @ \mathrm{Ho}$ nanostructures as a novel and highly efficient photocatalyst for degradation of organic pollution, J. Energy Chem. 26 (2017) 17-23. doi: 10.1016/j.jechem.2016.10.015.

43. M. Amiri, M. Salavati-Niasari, A. Akbari, A magnetic $\mathrm{CoFe}_{2} \mathrm{O}_{4} / \mathrm{SiO}_{2}$ nanocomposite fabricated by the sol-ge method for electrocatalytic oxidation and determination of L-cysteine, Microchim. Acta 184 (2017) 825-833. doi: 10.1007/s00604-016-2064-4.

44. M. Amiri, M. Salavati-Niasari, A. Pardakhty, M. Ahmadi, A. Akbari, Caffeine: A novel green precursor for synthesis of magnetic $\mathrm{CoFe}_{2} \mathrm{O}_{4}$ nanoparticles and $\mathrm{pH}$ sensitive magnetic alginate beads for drug delivery, Mater. Sci. Eng. C 76 (2017) 1085-1093. doi: 10.1016/j.msec.2017.03.208

45. A. Abbasi, D. Ghanbari, M. Salavati-Niasari, M. Hamadanian, Photo-degradation of methylene blue: photocatalyst and magnetic investigation of $\mathrm{Fe}_{2} \mathrm{O}_{3}-\mathrm{TiO}_{2}$ nanoparticles and nanocomposites, J. Mater. Sci. Mater. Electron. 27 (2016) 4800-4809. doi: 10.1007/s10854016-4361-4.

46. M. Amiri, A. Pardakhti, M. Ahmadi-Zeidabadi, A. Akbari, M. Salavati-Niasari, Magnetic nickel ferrite nanoparticles: Green synthesis by Urtica and therapeutic effect of frequency magnetic field on creating cytotoxic response in neural cell lines, Colloids Surf. B Biointerfaces 172 (2018) 244-253. doi: 10.1016/j.colsurfb.2018.08.049.

47. M. Amiri, A. Akbari, M. Ahmadi, A. Pardakhti, M. Salavati-Niasari, Synthesis and in vitro evaluation of a novel magnetic drug delivery system; proecological method for the preparation of $\mathrm{CoFe}_{2} \mathrm{O}_{4}$ nanostructures, J. Mol. Liq. 249 (2018) 1151-1160. doi: 10.1016/j.molliq.2017.11.133.

48. D. Ghanbari, M. Salavati-Niasari, Synthesis of urchin-like $\mathrm{CdS}-\mathrm{Fe} 3 \mathrm{O} 4$ nanocomposite and its application in flame retardancy of magnetic cellulose acetate, J. Ind. Eng. Chem. 24 (2015) 284-292. doi 10.1016/j.jiec.2014.09.043.

49. M. Amiri, T. Gholami, o. Amiri, A. Pardakhti, M. Ahmadi, A. Akbari, A. Amanatfard, M. Salavati-Niasari, The magnetic inorganic-organic nanocomposite based on $\mathrm{ZnFe}_{2} \mathrm{O}_{4}$-Imatinib-liposome for biomedical applications, in vivo and in vitro study, J. Alloys Compd. 849 (2020) 156604. doi: 10.1016/j.jallcom.2020.15660.

50. K. Zhu, Y. Ju, J. Xu, Z. Yang, S. Gao, Y. Hou, Magnetic nanomaterials: chemical design, synthesis, and potential applications, Acc. Chem. Res. 51 (2018) 404-413. doi:10.1021/acs.accounts.7b00407.

51. A. Gupta, R. Naregalkar, V. Vaidya, M. Gupta, Recent advances on surface engineering of magnetic iron oxide nanoparticles and their biomedical applications, $\begin{array}{lll}\text { Nanomedicine. } & 2 & \text { (2007) }\end{array}$ doi:10.2217/17435889.2.1.23.

52. K. Them, On magnetic dipole-dipole interactions of nanoparticles in magnetic particle imaging, Phys. Med. Biol. 62 (2017) 5623-5639. doi:10.1088/13616560/aa70ca.

53. D. Makovec, M. Drofenik, Non-stoichiometric zincferrite spinel nanoparticles, J. Nanoparticle Res. 10 (2008) 131-141. doi:10.1007/s11051-008-9400-5.

54. Hedman, D., \& Fjellström, M. (2013). Modeling the interactions between magnetic particles. Retrieved from http://urn.kb.se/resolve?urn=urn:nbn:se:Itu:diva-22908

55. D. Lisjak, P. Jenus, M. Drofenik, Formation of columnar structures by the magnetically directed assembly of cobalt ferrite nanoparticles, IEEE Trans. Magn. 48 (2012) 3303-3306. doi:10.1109/tmag.2012.2200659.

56. K. Bishop, C. Wilmer, S. Soh, B. Grzybowski, Nanoscale forces and their uses in self-assembly, Small. 5 (2009) 1600-1630. doi:10.1002/smll.200900358.

57. F. Leite, C. Bueno, A. Da Róz, E. Ziemath, O. Oliveira, Theoretical models for surface forces and adhesion and their measurement using atomic force microscopy, Int. J. Mol. Sci. 13 (2012) 12773-12856. doi:10.3390/ijms131012773.

58. W. Zhang, Nanoparticle aggregation: principles and modeling, Adv. Exp. Med. Biol. 811 (2014) 19-43. doi: 10.1007/978-94-017-8739-0 2

59. R. Tadmor, R. Rosensweig, J. Frey, J. Klein, Resolving the puzzle of ferrofluid dispersants, Langmuir. 16 (2000) 9117-9120. doi:10.1021/la0009137.

60. M. Chen, H. Shen, X. Li, J. Ruan, W. Yuan, Magnetic fluids' stability improved by oleic acid bilayer-coated structure via one-pot synthesis, Chem. Pap. 70 (2016) 1642-1648. doi:10.1515/chempap-2016-0096.

61. M. Bloemen, W. Brullot, T. Luong, N. Geukens, A. Gils, T. Verbiest, 2012. Improved functionalization of oleic acidcoated iron oxide nanoparticles for biomedical applications, J. Nanopart. Res. 14, 1100. doi:10.1007/s11051-012-1100-5.

62. M. Victory, R. Pant, S. Phanjoubam, 2020. Synthesis and characterization of oleic acid coated Fe-Mn ferrite based ferrofluid, Mater. Chem. Phys. 240, 122210. doi:10.1016/j.matchemphys.2019.122210.

63. M. Yu, S. Huang, K. Yu, A. Clyne, Dextran and polymer polyethylene glycol (PEG) coating reduce both 5 and 30 $\mathrm{nm}$ iron oxide nanoparticle cytotoxicity in 2D and 3D cell culture, Int. J. Mol. Sci. 13 (2012) 5554-5570. doi:10.3390/ijms13055554.

64. G. Kurlyandskaya, L. Litvinova, A. Safronov, V. Schupletsova, I. Tyukova, O. Khaziakhmatova, G. Slepchenko, K. Yurova, E. Cherempey, N. Kulesh, R. Andrade, I. Beketov, I. Khlusov, 2017. Water-based suspensions of iron oxide nanoparticles with electrostatic or steric stabilization by chitosan: fabrication, characterization and biocompatibility, Sensors. 17, 2605. doi:10.3390/s17112605.

65. A. Joseph, S. Mathew, Ferrofluids: Synthetic strategies, stabilization, physicochemical features, characterization, and applications, Chempluschem. 79 (2014) 1382-1420. doi:10.1002/cplu.201402202. 
66. R. Hong, S. Zhang, Y. Han, H. Li, J. Ding, Y. Zheng, Preparation, characterization and application of bilayer surfactant-stabilized ferrofluids, Powder Technol. 170 (2006) 1-11. doi:10.1016/j.powtec.2006.08.017.

67. N. Jain, Y. Wang, S. Jones, B. Hawkett, G. Warr, Optimized steric stabilization of aqueous ferrofluids and magnetic nanoparticles, Langmuir. 26 (2010) 4465-4472. doi:10.1021/la903513v.

68. C. Lin, C. Lee, W. Chiu, Preparation and properties of poly(acrylic acid) oligomer stabilized superparamagnetic ferrofluid, J. Colloid Interface Sci. 291 (2005) 411-420. doi:10.1016/j.jcis.2005.05.023.

69. A. Carvalho, J. Gallo, D. Pereira, P. Valentão, P. Andrade, L. Hilliou, P. Ferreira, M. Bañobre-López, J. Martins, 2019. Magnetic dehydrodipeptide-based self-assembled hydrogels for theragnostic applications, Nanomaterials. 9, 541. doi:10.3390/nano9040541.

70. S. Campelj, D. Makovec, M. Drofenik, 2008. Preparation and properties of water-based magnetic fluids, J. Phys. Condens. Matter. 20, 204101. doi:10.1088/09538984/20/20/204101.

71. A. Hajdú, E. Illés, E. Tombácz, I. Borbáth, Surface charging, polyanionic coating and colloid stability of magnetite nanoparticles, Colloids Surf. A Physicochem. Eng. $\quad$ Asp. $\quad 347 \quad$ (2009) 104-108. doi:10.1016/j.colsurfa.2008.12.039

72. Z. Wang, H. Zhu, X. Wang, F. Yang, X. Yang, 2009. Onepot green synthesis of biocompatible arginine-stabilized magnetic nanoparticles, Nanotechnology. 20, 465606. doi:10.1088/0957-4484/20/46/465606.

73. M. Amiri, M. Salavati-Niasari, A. Akbari. Magnetic nanocarriers: Evolution of spinel ferrites for medical applications, Adv. Colloid Interface Sci. 265 (2019) 2944. doi: /10.1016/j.cis.2019.01.003.

74. S. Mørup, M. Hansen, C. Frandsen, Magnetic interactions between nanoparticles, Beilstein J. $\begin{array}{lrr}\text { Nanotechnol. } & 1 & \text { (2010) } \\ \end{array}$ doi:10.3762/bjnano.1.22.

75. C. Vestal, Q. Song, Z. Zhang, Effects of interparticle interactions upon the magnetic properties of $\mathrm{CoFe}_{2} \mathrm{O}_{4}$ and $\mathrm{MnFe}_{2} \mathrm{O}_{4}$ nanocrystals, J. Phys. Chem. B 108 (2004) 18222-18227. doi:10.1021/jp0464526.

76. R. Fu, Y. Yan, C. Roberts, Z. Liu, Y. Chen, 2018. The role of dipole interactions in hyperthermia heating colloidal clusters of densely-packed superparamagnetic nanoparticles, Sci. Rep. 8, 4704. doi:10.1038/s41598018-23225-5.

77. L. Branquinho, M. Carrião, A. Costa, N. Zufelato, M. Sousa, R. Miotto, R. Ivkov, A. Bakuzis, 2013. Effect of magnetic dipolar interactions on nanoparticle heating efficiency: Implications for cancer hyperthermia, Sci. Rep. 3, 2887. doi:10.1038/srep02887.

78. R. Fu, Y. Yan, C. Roberts, 2015. Study of the effect of dipole interactions on hyperthermia heating the cluster composed of superparamagnetic nanoparticles, AIP Adv. 5, 127232. doi:10.1063/1.4939514.

79. A. Zubarev, L. Iskakova, Theory of physical properties of magnetic liquids with chain aggregates, J. Exp. Theor. Phys. 5 (1995) 857-866.

80. A. Zubarev, L. Iskakova, Effect of chainlike aggregates on dynamical properties of magnetic liquids, Phys. Rev. E 61 (2000) 5415-5421. doi:10.1103/physreve.61.5415

81. A. Zubarev, Rheological properties of polydisperse magnetic fluids. Effect of chain aggregates, J. Exp. Theor. Phys. 93 (2001) 80-88. doi:10.1134/1.1391522.

82. A. Abrikosov, S. Sacanna, A. Philipse, P. Linse, 2013. Selfassembly of spherical colloidal particles with offcentered magnetic dipoles, Soft Matter 9, 8904. doi:10.1039/c3sm27128e.
83. J. Wang, H. Dong, S. Li, 2018. Magnetic dipole-dipole interaction induced by the electromagnetic field, Phys. Rev. A 97, 013819. doi:10.1103/physreva.97.013819.

84. A. Mehdizadeh, R. Mei, J. Klausner, N. Rahmatian, Interaction forces between soft magnetic particles in uniform and non-uniform magnetic fields, Acta Mech. Sin. 26 (2010) 921-929. doi:10.1007/s10409-010-0383-y.

85. P. Sánchez, J. Cerdà, T. Sintes, A. Ivanov, S. Kantorovich, The effect of links on the interparticle dipolar correlations in supramolecular magnetic filaments, Soft Matter 11 (2015) 2963-2972. doi:10.1039/c5sm00172b.

86. D. Rozhkov, E. Pyanzina, E. Novak, J. Cerdà, T. Sintes, M. Ronti, P. Sánchez, K. Kantorovich. Self-assembly of polymer-like structures of magnetic colloids: Langevin dynamics study of basic topologies, Mol. Simulat. 44 (2017) 507-515. doi:10.1080/08927022.2017.1378815.

87. E. Novak, E. Pyanzina, S. Kantorovich, 2015. Behaviour of magnetic Janus-like colloids, J. Phys. Condens. Matter. 27, 234102. doi:10.1088/0953$8984 / 27 / 23 / 234102$.

88. P. Cremer, H. Löwen, A. Menzel, Superelastic stressstrain behavior in ferrogels with different types of magneto-elastic coupling, Phys. Chem. Chem. Phys. 18 (2016) 26670-26690. doi:10.1039/c6cp05079d.

89. R. Weeber, P. Kreissl, C. Holm, Studying the fieldcontrolled change of shape and elasticity of magnetic gels using particle-based simulations, Arch. Appl. Mech. 89 (2018) 3-16. doi:10.1007/s00419-018-1396-4.

90. R. Weeber, S. Kantorovich, C. Holm, 2015. Ferrogels cross-linked by magnetic particles: Field-driven deformation and elasticity studied using computer simulations, J. Chem. Phys. 143, 154901. doi:10.1063/1.4932371.

91. L. Rovigatti, S. Kantorovich, A. Ivanov, J. Tavares, F. Sciortino, 2013. Branching points in the lowtemperature dipolar hard sphere fluid, J. Chem. Phys. 139, 134901. doi:10.1063/1.4821935.

92. A. Abrikosov, S. Sacanna, A. Philipse, P. Linse, Selfassembly of spherical colloidal particles with offcentered magnetic dipoles, Soft Matter 9 (2013) 89048913. doi:10.1039/c3sm27128e.

93. A. Hynninen, M. Dijkstra, 2005. Phase diagram of dipolar hard and soft spheres: Manipulation of colloidal crystal structures by an external field, Phys. Rev. Lett. 94, 138303. doi:10.1103/physrevlett.94.138303.

94. M. Brito, M. Carignano, V. Marconi, 2020. Self-assembly of pseudo-dipolar nanoparticles at low densities and strong coupling, Sci. Rep. 10, 3971. doi:10.1038/s41598020-60417-4.

95. P. Santos, Z. Cao, J. Zhang, A. Alexander-Katz, R. Macfarlane, Dictating nanoparticle assembly via systems-level control of molecular multivalency, J. Am. Chem. Soc. $141 \quad$ (2019) 14624-14632. doi:10.1021/jacs.9b04999.

96. P. Santos, R. Macfarlane, Reinforcing supramolecular bonding with magnetic dipole interactions to assemble dynamic nanoparticle superlattices, J. Am. Chem. Soc. 142 (2020) 1170-1174. doi:10.1021/jacs.9b11476.

97. K. Yuet, D. Hwang, R. Haghgooie, P. Doyle, Multifunctional superparamagnetic Janus particles, Langmuir 26 (2010) 4281-4287. doi:10.1021/la903348s.

98. A. Rosa, F. Cunha, 2019. The influence of dipolar particle interactions on the magnetization and the rotational viscosity of ferrofluids, Phys. Fluids. 31, 052006. doi:10.1063/1.5093267.

99. E. Abenojar, S. Wickramasinghe, J. Bas-Concepcion, A. Samia, Structural effects on the magnetic hyperthermia properties of iron oxide nanoparticles, Prog. Nat. Sci. Mater. Int. $26 \quad$ (2016) 440-448. doi:10.1016/j.pnsc.2016.09.004. 
100.I. Obaidat, B. Issa, Y. Haik, Magnetic properties of magnetic nanoparticles for efficient hyperthermia, Nanomaterials $\quad 5 \quad$ (2015) 63-89. doi:10.3390/nano5010063.

101. A. Hervault, N. Thanh, Magnetic nanoparticle-based therapeutic agents for thermo-chemotherapy treatment of cancer, Nanoscale 6 (2014) 11553-11573. doi:10.1039/c4nr03482a.

102. Z. Zhang, S. Song, Thermosensitive/superparamagnetic iron oxide nanoparticle-loaded nanocapsule hydrogels for multiple cancer hyperthermia, Biomaterials 106 (2016) 13-23.

103. Z. Hedayatnasab, F. Abnisa, W. Daud, Review on magnetic nanoparticles for magnetic nanofluid hyperthermia application, Mater. Design 123 (2017) 174-196. doi:10.1016/j.matdes.2017.03.036

104. A. Bhattacharya, R. Mahajan, Temperature dependence of thermal conductivity of biological tissues, Physiol. Meas. 24 (2003) 769-783. doi:10.1088/0967$3334 / 24 / 3 / 312$.

105. M. Dewhirst, B. Viglianti, M. Lora-Michiels, M. Hanson, P. Hoopes, Basic principles of thermal dosimetry and thermal thresholds for tissue damage from hyperthermia, Int. J. Hyperther. 19 (2003) 267-294. doi:10.1080/0265673031000119006.

106. P. Yarmolenko, E. Moon, C. Landon, A. Manzoor, D. Hochman, B. Viglianti, M. Dewhirst, Thresholds for thermal damage to normal tissues: An update, Int. J. Hyperther. $27 \quad$ (2011) 320-343. doi:10.3109/02656736.2010.534527.

107. U. Engelmann, A. Roeth, D. Eberbeck, E. Buhl, U. Neumann, T. Schmitz-Rode, I. Slabu, 2019. Combining bulk temperature and nanoheating enables advanced magnetic fluid hyperthermia efficacy on pancreatic tumor cells, Sci. Rep. 8, 13210. doi:10.1038/s41598-01831553-9.

108. X. Tang, Y. Xu, J. Chen, T. Ying, L. Wang, L. Jiang, Y. Wang, Z. Wang, Y. Ling, F. Wang, L. Yao, H. Ran, Z. Wang, B. Hu, Y. Zheng, Intermittent time-set technique controlling the temperature of magnetic-hyperthermiaablation for tumor therapy, RSC Adv. 8 (2018) 16410 16418. doi:10.1039/c8ra01176a.

109. G. Landi, 2014. Role of dipolar interaction in magnetic hyperthermia, Phys. Rev. B. 89, 014403. doi:10.1103/physrevb.89.014403.

110. A. Pereira, C. Pereira, A. Silva, D. Schmool, C. Freire, J. Grenèche, J. Araújo, 2011. Unravelling the effect of interparticle interactions and surface spin canting in $\gamma$ $\mathrm{Fe}_{2} \mathrm{O}_{3} @ \mathrm{SiO}_{2}$ superparamagnetic nanoparticles, J. Appl. Phys. 109, 114319. doi:10.1063/1.3583652.

111.D. Serantes, D. Baldomir, C. Martinez-Boubeta, K. Simeonidis, M. Angelakeris, E. Natividad, M. Castro, A. mediano, D. Chen, A. Sanchez, LI. Calcells, B. Martínez, 2010. Influence of dipolar interactions on hyperthermia properties of ferromagnetic particles, J. Appl. Phys. 108 , 073918. doi:10.1063/1.3488881.

112. M. Palihawadana-Arachchige, H. Nemala, V. Naik, R. Naik, 2017. Effect of magnetic dipolar interactions on temperature dependent magnetic hyperthermia in ferrofluids, J. Appl. Phy. 121, 023901. doi:10.1063/1.4973879.

113. A. Giustini, R. Ivkov, P. Hoopes, 2011. Magnetic nanoparticle biodistribution following intratumoral administration, Nanotechnology 22, 345101. doi:10.1088/0957-4484/22/34/345101.

114. B. Mehdaoui, R. Tan, A. Meffre, J. Carrey, S. Lachaize, B. Chaudret, M. Respaud, 2013. Increase of magnetic hyperthermia efficiency due to dipolar interactions in low-anisotropy magnetic nanoparticles: Theoretical and experimental results, Phys. Rev. B 87, 174419. doi:10.1103/physrevb.87.174419.

115. D. Niculaes, A. Lak, G. Anyfantis, S. Marras, O. Laslett, S Avugadda, M. Cassani, D. Serantes, O. Hovorka, R. Chantrell, T. Pellegrino, Asymmetric assembling of iron oxide nanocubes for improving magnetic hyperthermia performance, ACS Nano 11 (2017) 12121-12133.

116. E. Périgo, G. Hemery, O. Sandre, D. Ortega, E. Garaio, F. Plazaola, F. Teran, 2015. Fundamentals and advances in magnetic hyperthermia, Appl. Phys. Rev. 2, 041302. doi:10.1063/1.4935688.

117. A. Abu-Bakr, A. Zubarev, 2020. On the theory of magnetic hyperthermia: clusterization of nanoparticles, Phil. Trans. R. Soc. A 378, 20190251. doi:10.1098/rsta.2019.0251.

118. O. Wichterle, D. Lím, Hydrophilic gels for biological use, Nature 185 (1960) 117-118. doi:10.1038/185117a0

119. J. Yang, R. Bai, B. Chen, Z. Suo, 2019. Hydrogel adhesion: A supramolecular synergy of chemistry, topology, and mechanics, Adv. Funct. Mater. 30, 1901693. doi:10.1002/adfm.201901693.

120. K. Lee, D. Mooney, Hydrogels for tissue engineering, Chem. Rev. $101 \quad$ (2001) 1869-1880. doi:10.1021/cr000108x.

121. J. Koffler, W. Zhu, X. Qu, O. Platoshyn, J. Dulin, J. Brock, L. Graham, P. Lu, J. Sakamoto, M. Marsala, S. Chen, M. Tuszynki, Biomimetic 3D-printed scaffolds for spinal cord injury repair, Nat. Med. 25 (2019) 263-269. doi:10.1038/s41591-018-0296-z.

122. J. Burdick, K. Anseth, Photoencapsulation of osteoblasts in injectable RGD-modified PEG hydrogels for bone tissue engineering, Biomaterials 23 (2002) 4315-4323.

123. K. Stevens, J. Miller, B. Blakely, C. Chen, S. Bhatia, Degradable hydrogels derived from PEG-diacrylamide for hepatic tissue engineering, J. Biomed. Mater. Res. A 103 (2015) 3331-3338.

124. J. Tavakoli, Y. Tang, 2017. Hydrogel based sensors for biomedical applications: An updated review, Polymers 9, 364. doi:10.3390/polym9080364.

125. I. Jung, J. Kim, B. Choi, K. Lee, H. Lee, 2017. Hydrogel based biosensors for in vitro diagnostics of biochemicals, proteins, and genes, Adv. Healthc. Mater. 6, 1601475. doi:10.1002/adhm. 201601475.

126. A. Bashari, A. Rouhani Shirvan, M. Shakeri, Cellulosebased hydrogels for personal care products, Polym. Adv. Technol. 29 (2018) 2853-2867. doi:10.1002/pat.4290.

127. W. Liechty, R. Scheuerle, J. Ramirez, N. Peppas, Uptake and function of membrane-destabilizing cationic nanogels for intracellular drug delivery, Bioeng Transl Med. 4 (2019) 17-29.

128. M. Hamidi, A. Azadi, P. Rafiei, Hydrogel nanoparticles in drug delivery, Adv. Drug Deliv. Rev. 60 (2008) 16381649. doi:10.1016/j.addr.2008.08.002.

129. A. Childs, H. Li, D. Lewittes, B. Dong, W. Liu, X. Shu, C. Sun, H. Zhang, 2016. Fabricating customized hydrogel contact lens, Sci. Rep. 6, 34905. doi:10.1038/srep34905.

130.P. Nicolson, J. Vogt, Soft contact lens polymers: an evolution, Biomaterials 22 (2001) 3273-3283. doi:10.1016/s0142-9612(01)00165-x.

131. T. Hoare, D. Kohane, Hydrogels in drug delivery: Progress and challenges, Polymer, 49 (2008) 1993-2007. doi: 10.1016/j.polymer.2008.01.027.

132. K. Varaprasad, G. Raghavendra, T. Jayaramudu, M. Yallapu, R. Sadiku, A mini review on hydrogels classification and recent developments in miscellaneous applications, Mater. Sci. Eng. C 79 (2017) 958-971. doi: 10.1016/j.msec.2017.05.096

133. E. Caló, V. Khutoryanskiy, Biomedical applications of hydrogels: A review of patents and commercial products, Eur. Polym. J. 65 (2015) 252-267. 
134. R. Selegård, C. Aronsson, C. Brommesson, S. Dånmark, D. Aili, 2017. Folding driven self-assembly of a stimuliresponsive peptide-hyaluronan hybrid hydrogel, Sci. Rep. 7, 7013. doi:10.1038/s41598-017-06457-9.

135. M. Ma, Y. Kuang, Y. Gao, Y. Zhang, P. Gao, B. Xu, Aromatic-Aromatic interactions induce the selfassembly of pentapeptidic derivatives in water to form nanofibers and supramolecular hydrogels, J. Am. Chem. Soc. 132 (2010) 2719-2728. doi:10.1021/ja9088764.

136. S. Salatin, J. Barar, M. Barzegar-Jalali, K. Adibkia, M. Milani, M. Jelvehgari, Hydrogel nanoparticles and nanocomposites for nasal drug/vaccine delivery, Arch. Pharmacal Res. 39 (2016) 1181-1192. doi:10.1007/s12272-016-0782-0.

137. F. Ullah, M. Othman, F. Javed, Z. Ahmad, H. Akil, Classification, processing and application of hydrogels: A review, Mater. Sci. Eng. C. 57 (2015) 414-433. doi:10.1016/j.msec.2015.07.053.

138. M. Davidovich-Pinhas, H. Bianco-Peled, Alginate-PEGAc: A new mucoadhesive polymer, Acta Biomaterialia. 7 (2011) 625-633. doi:10.1016/j.actbio.2010.09.021.

139. L. Chen, S. Revel, K. Morris, L. Serpell, D. Adams, Effect of molecular structure on the properties of naphthalene-dipeptide hydrogelators, Langmuir 26 (2010) 13466-13471. doi:10.1021/la102059x.

140. T. Lorson, S. Jaksch, M. Lübtow, T. Jüngst, J. Groll, T. Lühmann, R. Luxenhofer, A Thermogelling supramolecular hydrogel with sponge-like morphology as a cytocompatible bioink, Biomacromolecules 18 (2017) 2161-2171. doi:10.1021/acs.biomac.7b00481.

141.J. Sun, H. Tan, Alginate-based biomaterials for regenerative medicine applications, Materials 6 (2013) 1285-1309. doi: 10.3390/ma6041285

142. K. Pal, A. Banthia, D. Majumdar, Polymeric hydrogels: characterization and biomedical applications, Des. Monomers Polym. 12 (2009) 197-220. doi:10.1163/156855509x436030.

143. Du, X., Zhou, J., Shi J. and Xu, B. (2015). Supramolecular hydrogelators and hydrogels: From soft matter to molecular biomaterials. Chem. Rev., 115, 13165-13307.

144. M. McKenzie, D. Betts, A. Suh, K. Bui, L. Kim, H. Cho, Hydrogel-based drug delivery systems for poorly watersoluble drugs, Molecules 20 (2015) 20397-20408. doi:10.3390/molecules201119705.

145.J. Li, D. Mooney, 2016. Designing hydrogels for controlled drug delivery, Nat. Rev. Mater. 1, 16071. doi:10.1038/natrevmats.2016.71.

146. H. Vilaça, A. Hortelão, E. Castanheira, M. Queiroz, L. Hilliou, I. Hamley, J. Martins, P. Ferreira, Dehydrodipeptide hydrogelators containing naproxen $n$ capped tryptophan: Self-assembly, hydrogel characterization, and evaluation as potential drug nanocarriers, Biomacromolecules 16 (2015) 3562-3573. doi:10.1021/acs.biomac.5b01006.

147. M. Antman-Passig, O. Shefi, Remote magnetic orientation of 3D collagen hydrogels for directed neuronal regeneration, Nano Lett. 16 (2016) 2567-2573. doi:10.1021/acs.nanolett.6b00131.

148. A. Jonker, D. Löwik, J. van Hest, Peptide- and proteinbased hydrogels, Chem. Mater. 24 (2012) 759-773. doi:10.1021/cm202640w.

149. A. Cardoso, A. Alvarez Alvarez, B. Cattoz, P. Griffiths, S. King, W. Frith, D. Adams, The influence of the kinetics of self-assembly on the properties of dipeptide hydrogels, Faraday Discuss. $166 \quad$ (2013) 101-116. doi:10.1039/c3fd00104k.

150. G. Fichman, E. Gazit, Self-assembly of short peptides to form hydrogels: Design of building blocks, physical properties and technological applications, Acta
Biomater.

10

doi:10.1016/j.actbio.2013.08.013.

1671-1682.

151. C. Gonçalves, P. Pereira, M. Gama, Self-assembled hydrogel nanoparticles for drug delivery applications, Materials 3 (2010) 1420-1460. doi:10.3390/ma3021420.

152. R. Dong, Y. Pang, Y. Su, X. Zhu, Supramolecular hydrogels: synthesis, properties and their biomedical applications, Biomater. Sci. 3 (2015) 937-954. doi:10.1039/c4bm00448e.

153.S. Koutsopoulos, Self-assembling peptide nanofiber hydrogels in tissue engineering and regenerative medicine: Progress, design guidelines, and applications, J. Biomed. Mater. Res. A. 104 (2016) 1002-1016. doi:10.1002/jbm.a.35638.

154. Sutton, S., Campbell, N., Cooper, A., Kirkland, M., Frith, W. and Adams, D. (2009). Controlled release from modified amino acid hydrogels governed by molecular size or network dynamics. Langmuir, 25, 10285-10291.

155. J. Lim, Q. Lin, K. Xue, X. Loh, 2019. Recent advances in supramolecular hydrogels for biomedical applications, Mater. Today Adv. 3, 100021. doi:10.1016/j.mtadv.2019.100021.

156. Y. Li, F. Wang, H. Cui, Peptide-based supramolecular hydrogels for delivery of biologics, Bioengineering \& Transla. Med. 1 (2016) 306-322. doi:10.1002/btm2.10041.

157. J. Xu, M. Tam, S. Samaei, S. Lerouge, J. Barralet, M. Stevenson, M. Cerruti, Mucoadhesive chitosan hydrogels as rectal drug delivery vessels to treat ulcerative colitis, Acta Biomater. 48 (2017) 247-257. doi:10.1016/j.actbio.2016.10.026.

158. A. Mandal, J. Clegg, A. Anselmo, 2020. S. Mitragotri, Hydrogels in the clinic, Bioengineering \& Transla. Med. 5, e10158. doi:10.1002/btm2.10158.

159. R. Narayanaswamy, V. Torchilin, 2019. Hydrogels and their applications in targeted drug delivery, Molecules 24, 603. doi:10.3390/molecules24030603.

160.G. Patel, C. Dalwadi, Recent patents on stimuli responsive hydrogel drug delivery system, Recent. Pat. Drug Deliv. Formul. 7 (2013) 206-215. doi:10.2174/1872211307666131118141600.

161. N. Bhattarai, J. Gunn, M. Zhang, Chitosan-based hydrogels for controlled, localized drug delivery, Adv. Drug Deliv. Rev. $62 \quad$ (2010) 83-99. doi:10.1016/j.addr.2009.07.019

162.S. Bidarra, C. Barrias, P. Granja, Injectable alginate hydrogels for cell delivery in tissue engineering, Acta Biomaterialia $10 \quad$ (2014) 1646-1662. Doi: 10.1016/j.actbio.2013.12.006

163. E. Popa, P. Carvalho, A. Dias, T. Santos, V. Santo, A. Marques, C. Viegas, I. Dias, M. Gomes, R. Reis, Evaluation of the in vitro and in vivo biocompatibility of carrageenan-based hydrogels. J. Biomed. Mater. Res. A 102 (2014) 4087-4097. Doi: 10.1002/jbm.a.35081

164. L. Han, J. Xu, X. Lu, D. Gan, Z. Wang, K. Wang, H. Zhang, H. Yuan, J. Weng, Biohybrid methacrylated gelatin/polyacrylamide hydrogels for cartilage repair, J. Mater. Chem. B 5 (2017) 731-741. Doi: 10.1039/C6TB02348G

165. H. Mansur, E. de S. Costa, A. Mansur, E. BarbosaStancioli, Cytocompatibility evaluation in cell-culture systems of chemically crosslinked chitosan/PVA hydrogels, Mater. Sci. Eng. C 29 (2009) 1574-1583. Doi: 10.1016/j.msec.2008.12.012

166. M. Husain, A. Gupta, B. Alashwal, S. Sharma, Synthesis of PVA/PVP based hydrogel for biomedical applications: a review, Energy Sources Part A 40 (2018) 2388-2393. Doi: 10.1080/15567036.2018.1495786

167. L. Suggs, M. Shive, C. Garcia, J. Anderson, A. Mikos, In vitro cytotoxicity and in vivo biocompatibility of 
poly(propylene fumarate-co-ethylene glycol) hydrogels, J. Biomed. Mater. Res. 46 (1999) 22-32. Doi: 10.1002/(sici)1097-4636(199907)46:1<22::aidjbm3>3.0.co;2-r

168. T. Lopez-Silva, D. Leach, A. Azares, I. Li, D. Woodside, J. Hartgerink, Chemical functionality of multidomain peptide hydrogels governs early host immune response. Biomaterials $231 \quad$ (2020) 119667-11980. Doi: 10.1016/j.biomaterials.2019.119667

169. S. Mondal, S. Das, A. Nandi, A review on recent advances in polymer and peptide hydrogels, Soft Matter 16 (2020) 1404-1454. doi:10.1039/c9sm02127b.

170. S. Buwalda, T. Vermonden, W. Hennink, hydrogels for therapeutic delivery: Current developments and future directions, Biomacromolecules 18 (2017) 316-330. doi:10.1021/acs.biomac.6b01604

171. R. Weeber, S. Kantorovich, C. Holm, Ferrogels crosslinked by magnetic nanoparticles-Deformation mechanisms in two and three dimensions studied by means of computer simulations, J. Magn. Magn. Mater. 383 (2015) 262-266. doi:10.1016/j.jmmm.2015.01.018.

172. V. Nguyen, A. Ahmed, R. Ramanujan, Morphing soft magnetic composites, Adv. Mater. 24 (2012) 4041-4054. doi:10.1002/adma.201104994.

173. R. Ramanujan, L. Lao, The mechanical behavior of smart magnet-hydrogel composites, Smart Mater. Struct. 15 (2006) 952-956. doi:10.1088/0964-1726/15/4/008.

174. T. Mitsumata, Y. Horikoshi, K. Negami, High-Power actuators made of two-phase magnetic gels, Jpn. J. Appl. Phys. 47 (2008) 7257-7261. doi:10.1143/jjap.47.7257.

175. H. Meng, G. Li, Reversible switching transitions of stimuli-responsive shape changing polymers, J. Mater. Chem. A. 1 (2013) 7838-7865. doi:10.1039/c3ta10716g.

176. M. Shin, S. Kim, S. Kim, S. Park, Y. Hyun, Y. Lee, K. Lee, S. Han, D. Jang, Y. Kim, Z. Cho, I. So, G. Spinks, Controlled magnetic nanofiber hydrogels by clustering ferritin, Langmuir. $24 \quad$ (2008) 12107-12111. doi:10.1021/la802155a.

177. Y. Liang, L. Zhang, W. Jiang, W. Li, Embedding magnetic nanoparticles into polysaccharide-based hydrogels for magnetically assisted bioseparation, Chemphyschem. 8 (2007) 2367-2372. doi:10.1002/cphc.200700359.

178. J. Sang, R. Wu, P. Guo, J. Du, S. Xu, J. Wang, 2015. Affinity-tuned peroxidase-like activity of hydrogelsupported $\mathrm{Fe}_{3} \mathrm{O}_{4}$ nanozyme through alteration of crosslinking concentration, J. Appl. Polym. Sci. 133, 43065. doi:10.1002/app.43065.

179. M. Witt, S. Hinrichs, N. Möller, S. Backes, B. Fischer, R. von Klitzing, Distribution of $\mathrm{CoFe}_{2} \mathrm{O}_{4}$ nanoparticles inside PNIPAM-based microgels of different cross-linker distributions, J. Phys. Chem. B. 123 (2019) 2405-2413. doi:10.1021/acs.jpcb.8b09236.

180. E. Mikhnevich, P. Chebotkova, A. Safronov, G. Kurlyandskaya, 2019. Influence of uniform magnetic field on elastic modulus in polyacrylamide ferrogels with embedded nickel nanoparticles, J. Phys. Conf. Ser. 1389, 012059. doi:10.1088/1742-6596/1389/1/012059.

181. L. Roeder, M. Reckenthäler, L. Belkoura, S. Roitsch, R. Strey, A. Schmidt, Covalent ferrohydrogels based on elongated particulate cross-linkers, Macromolecules. 47 (2014) 7200-7207. doi:10.1021/ma501396j.

182.Z. Liu, J. Liu, X. Cui, X. Wang, L. Zhang, P. Tang, 2020. Recent advances on magnetic sensitive hydrogels in tissue engineering, Front. Chem. 8, 124 doi:10.3389/fchem.2020.00124.

183. J. Zhang, Q. Huang, J. Du, Recent advances in magnetic hydrogels, Polym. Int. 65 (2016) 1365-1372. doi:10.1002/pi.5170.

184. S. Veloso, C. Magalhães, A. Rodrigues, H. Vilaça, M. Queiroz, J. Martins et al., Novel dehydropeptide-based magnetogels containing manganese ferrite nanoparticles as antitumor drug nanocarriers, Phys. Chem. Chem. Phys. 21 (2019) 10377-10390. doi:10.1039/c9cp00352e.

185. P. Das, S. Yuran, J. Yan, P. Lee, M. Reches, Sticky tubes and magnetic hydrogels co-assembled by a short peptide and melanin-like nanoparticles, Chem. $\begin{array}{llll}\text { Commun. } & 51 & \text { (2015) 5432-5435. }\end{array}$ doi:10.1039/c4cc07671k.

186. J. Huang, Y. Xue, N. Cai, H. Zhang, K. Wen, X. Luo et al., Efficient reduction and $\mathrm{pH}$ co-triggered DOX-loaded magnetic nanogel carrier using disulfide crosslinking, Mater. Sci. Eng. C. $46 \quad$ (2015) 41-51. doi:10.1016/j.msec.2014.10.003.

187. M. Lin, D. Lu, J. Zhu, C. Yang, Y. Zhang, Z. Liu, 2012. Magnetic enzyme nanogel (MENG): a universal synthetic route for biocatalysts, Chem. Commun. 48, 3315. doi:10.1039/c2cc30189j.

188. R. Turcu, V. Socoliuc, I. Craciunescu, A. Petran, A. Paulus, M. Franzreb et al., Magnetic microgels, a promising candidate for enhanced magnetic adsorbent particles in bioseparation: synthesis, physicochemical characterization, and separation performance, Soft Matter. 11 (2015) 1008-1018. doi:10.1039/c4sm02430c.

189. A. Seyfoori, S. Seyyed Ebrahimi, E. Samiei, M. Akbari, multifunctional hybrid magnetic microgel synthesis for immune-based isolation and post-isolation culture of tumor cells, ACS Appl. Mater. Interfaces. 11 (2019) 24945-24958. doi:10.1021/acsami.9b02959.

190. S. Lee, Y. Park, J. Kim, 2018. Thermoresponsive behavior of magnetic nanoparticle complexed pNIPAm-co-AAc microgels, Appl. Sci. 8, 1984. doi:10.3390/app8101984.

191. Y. Zhang, B. Yang, X. Zhang, L. Xu, L. Tao, S. Li, Y. Wei, A magnetic self-healing hydrogel, Chem. Commun. 48 (2012) 9305-9307. doi:10.1039/c2cc34745h.

192. D. Meira, R. Domingues, M. Rodrigues, E. Alves, N. Barradas, J. Borges, F. Vaz, 2020. Thin films of Au-Al2O3 for plasmonic sensing, Appl. Surf. Sci. 500, 144035. doi:10.1016/j.apsusc.2019.144035.

193. S. Almohammed, M. Alruwaili, E. Reynaud, G. Redmond, J. Rice, B. Rodriguez, 3D-Printed peptide-hydrogel nanoparticle composites for surface-enhanced raman spectroscopy sensing, ACS Appl. Nano Mater. 2 (2019) 5029-5034. doi:10.1021/acsanm.9b00940.

194.Y. Yeh, B. Creran, V. Rotello, Gold nanoparticles: preparation, properties, and applications in bionanotechnology, Nanoscale. 4 (2012) 1871-1880. doi:10.1039/c1nr11188d.

195.G. Kikelbick, Hybrid Materials: Synthesis, Characterization, and Applications, first ed., Wiley, Weinheim, 2007.

196. J. Qin, I. Asempah, S. Laurent, A. Fornara, R. Muller, M. Muhammed, Injectable superparamagnetic ferrogels for controlled release of hydrophobic drugs, Adv. Mater. 21 (2009) 1354-1357. doi:10.1002/adma.200800764.

197. A. Ryzhkov, P. Melenev, C. Holm, Y. Raikher, Coarsegrained molecular dynamics simulation of small ferrogel objects, J. Magn. Magn. Mater. 383 (2015) 277-280. doi:10.1016/j.jmmm.2014.11.008.

198. A. Menzel, 2014. Bridging from particle to macroscopic scales in uniaxial magnetic gels, J. Chem. Phys. 141, 194907. doi:10.1063/1.4901275.

199. A. Ryzhkov, P. Melenev, M. Balasoiu, Y. Raikher, Structure organization and magnetic properties of microscale ferrogels: The effect of particle magnetic anisotropy, J. Chem. Phys. 145 (2016) 074905. doi:10.1063/1.4961299.

200. A. Attaran, J. Brummund, T. Wallmersperger, Development of a continuum model for ferrogels, J. 
Intel. Mat. Syst. Str. 28 (2016) 1358-1375. doi:10.1177/1045389x16672564.

201. A. Attaran, J. Brummund, T. Wallmersperger, Modeling and finite element simulation of the magnetomechanical behavior of ferrogels, J. Magn. Magn. Mater. 431 (2017) 188-191. doi:10.1016/j.jmmm.2016.09.058.

202.S. Bohlius, H. Brand, H. Pleiner, 2004. Macroscopic dynamics of uniaxial magnetic gels, Phys. Rev. E. 70. 061411. doi:10.1103/physreve.70.061411.

203. D. Madeo, G. Bevilacqua, V. Biancalana, Y. Dancheva, C. Mocenni, 2020. A physical model for the characterization of magnetic hydrogels subject to external magnetic fields, J. Magn. Magn. Mater. 493, 165674. doi:10.1016/j.jmmm.2019.165674.

204.S. Goh, R. Wittmann, A. Menzel, H. Löwen, 2019. Classical density functional theory for a two-dimensional isotropic ferrogel model with labeled particles, Phys. Rev. E. 100, 012605. doi:10.1103/physreve.100.012605.

205. S. Goh, A. Menzel, H. Löwen, Dynamics in a onedimensional ferrogel model: relaxation, pairing, shockwave propagation, Phys. Chem. Chem. Phys. 20 (2018) 15037-15051. doi:10.1039/c8cp01395k.

206. P. Gebhart, T. Wallmersperger, A general framework for the modeling of porous ferrogels at finite strains, J. Mech. Phys. Solids. 122 (2019) 69-83. doi:10.1016/j.jmps.2018.08.001.

207. M. Lopez-Lopez, D. Borin, A. Zubarev, 2017. Shear elasticity of isotropic magnetic gels, Phys. Rev. E. 96, 022605. doi:10.1103/physreve.96.022605.

208. D. Borin, D. Chirikov, A. Zubarev, 2018. Shear elasticity of magnetic gels with internal structures, Sensors. 18, 2054. doi:10.3390/s18072054.

209. C. Gila-Vilchez, J. Duran, F. Gonzalez-Caballero, A. Zubarev, M. Lopez-Lopez, 2019, Magnetorheology of alginate ferrogels, Smart Mater. Str. 28, 035018. doi:10.1088/1361-665x/aafeac.

210. P. Melenev, A. Ryzhkov, 2019. Simulation of uniaxial deformation of a ferrogel sample exposed by the external magnetic field, IOP Conf. Ser. Mater Sci. Eng. 581, 012039. doi:10.1088/1757-899x/581/1/012039.

211. S. Huang, G. Pessot, P. Cremer, R. Weeber, C. Holm, J. Nowak, S. Odenbach, A. Menzel, G. Auernhammer, Buckling of paramagnetic chains in soft gels, Soft Matter. 12 (2016) 228-237. doi:10.1039/c5sm01814e.

212. G. Pessot, R. Weeber, C. Holm, H. Löwen, A. Menzel, 2015. Towards a scale-bridging description of ferrogels and magnetic elastomers, J. Phys. Condens. Mat. 27, 325105. doi:10.1088/0953-8984/27/32/325105.

213. A. Zubarev, On the theory of the magnetic deformation of ferrogels, Soft Matter. 8 (2012) 3174-3179. doi:10.1039/c2sm06961j.

214.D. Wood, P. Camp, 2011. Modeling the properties of ferrogels in uniform magnetic fields, Phys. Rev. E. 83, 011402. doi:10.1103/physreve.83.011402.

215. G. Pessot, H. Löwen, A. Menzel, 2016. Dynamic elastic moduli in magnetic gels: Normal modes and linear response, J. Chem. Phys. 145, 104904. doi:10.1063/1.4962365.

216. G. Pessot, P. Cremer, D. Borin, S. Odenbach, H. Löwen, A. Menzel, 2014. Structural control of elastic moduli in ferrogels and the importance of non-affine deformations, J. Chem. Phys. 141, 124904 doi:10.1063/1.4896147.

217. P. Cremer, M. Heinen, A. Menzel, H. Löwen, 2017. A density functional approach to ferrogels, J. Phys. Condens. Mat. 29, 275102. doi:10.1088/1361 $648 \mathrm{x} / \mathrm{aa} 73 \mathrm{bd}$

218. E. Minina, P. Sánchez, C. Likos, S. Kantorovich, The influence of the magnetic filler concentration on the properties of a microgel particle: Zero-field case, J.
Magn. Magn. Mater. 459 doi:10.1016/j.jmmm.2017.10.107.

219. S. Backes, M. Witt, E. Roeben, L. Kuhrts, S. Aleed, A. Schmidt, R. Klitzing, Loading of PNIPAM based microgels with $\mathrm{CoFe}_{2} \mathrm{O}_{4}$ nanoparticles and their magnetic response in bulk and at surfaces, J. Phys. Chem. B. 119 (2015) 12129-12137. doi:10.1021/acs.jpcb.5b03778.

220. R. Weeber, S. Kantorovich, C. Holm, Deformation mechanisms in 2D magnetic gels studied by computer simulations, Soft Matter. 8 (2012) 9923-9932. doi:10.1039/c2sm26097b.

221.S. Monz, A. Tschöpe, R. Birringer, 2008. Magnetic properties of isotropic and anisotropic $\mathrm{CoFe}_{2} \mathrm{O}_{4}$-based ferrogels and their application as torsional and rotational actuators, Phys. Rev. E. 78, 021404. doi:10.1103/physreve.78.021404.

222. A. Zubarev, D. Chirikov, D. Borin, G. Stepanov, Hysteresis of the magnetic properties of soft magnetic gels, Soft Matter. 12 (2016) 6473-6480. doi:10.1039/c6sm01257d.

223. D. Romeis, V. Toshchevikov, M. Saphiannikova, Effects of local rearrangement of magnetic particles on deformation in magneto-sensitive elastomers, Soft Matter. 15 (2019) 3552-3564. doi:10.1039/c9sm00226j.

224. Weeber, R., Holm, C. Interplay between particle microstructure, network topology and sample shape in magnetic gels-a molecular dynamics simulation study. ArXiv preprint arXiv:1704.06578 (2017)

225. L. Fischer, A. Menzel, 2019. Magnetostriction in magnetic gels and elastomers as a function of the internal structure and particle distribution, J. Chem. Phys. 151, 114906. doi:10.1063/1.5118875.

226. A. Zubarev, Magnetodeformation of ferrogels and ferroelastomers. Effect of microstructure of the particles' spatial disposition, Physica A 392 (2013) 48244836. doi:10.1016/j.physa.2013.06.054.

227. G. Filipcsei, M. Zrínyi, 2010. Magnetodeformation effects and the swelling of ferrogels in a uniform magnetic field, J. Phys. Condens. Mat. 22, 276001. doi:10.1088/0953-8984/22/27/276001.

228. Q. Liu, H. Li, K. Lam, Optimization of the cell microenvironment in a dual magnetic- $\mathrm{pH}$-sensitive hydrogel-based scaffold by multiphysics modeling,

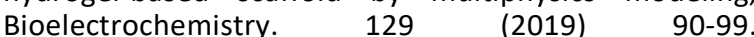
doi:10.1016/j.bioelechem.2019.05.004.

229. K. Dashnyam, R. Perez, R. Singh, E. Lee, H. Kim, Hybrid magnetic scaffolds of gelatin-siloxane incorporated with magnetite nanoparticles effective for bone tissue engineering, RSC Adv. 4 (2014) 40841-40851. doi:10.1039/c4ra06621a.

230. X. Zhao, J. Kim, C. Cezar, N. Huebsch, K. Lee, K. Bouhadir, D. Mooney, Active scaffolds for on-demand drug and cell delivery, Proc. Natl. Acad. Sci. 108 (2010) 67-72. doi:10.1073/pnas.1007862108.

231. M. Babincová, D. Leszczynska, P. Sourivong, P. Čičmanec, P. Babinec, Superparamagnetic gel as a novel material for electromagnetically induced hyperthermia, J. Magn. Magn. Mater. 225 (2001) 109-112. doi:10.1016/s0304-8853(00)01237-3.

232. L. Lao, R. Ramanujan, Magnetic and hydrogel composite materials for hyperthermia applications, J. Mater. Sci. Mater. Med. $15 \quad$ (2004) 1061-1064. doi:10.1023/b:jmsm.0000046386.78633.e5.

233. T. Liu, S. Hu, T. Liu, D. Liu, S. Chen, Magnetic-sensitive behavior of intelligent ferrogels for controlled release of drug, Langmuir. $22 \quad$ (2006) 5974-5978. doi:10.1021/la060371e.

234.S. Hu, T. Liu, D. Liu, S. Chen, Controlled pulsatile drug release from a ferrogel by a high-frequency magnetic field, Macromolecules. 40 (2007) 6786-6788. doi:10.1021/ma0707584 
235. O. Savchak, T. Morrison, K. Kornev, O. Kuksenok, Controlling deformations of gel-based composites by electromagnetic signals within the $\mathrm{GHz}$ frequency range, Soft Matter. $14 \quad$ (2018) 8698-8708. doi:10.1039/c8sm01207e.

236. S. Potorac, M. Popa, V. Maier, G. Lisa, L. Verestiuc, New hydrogels based on maleilated collagen with potential applications in tissue engineering, Mater. Sci. Eng. C. 32 (2012) 236-243. doi:10.1016/j.msec.2011.10.024.

237. L. Moreira Teixeira, J. Feijen, C. van Blitterswijk, P. Dijkstra, M. Karperien, Enzyme-catalyzed crosslinkable hydrogels: Emerging strategies for tissue engineering, Biomaterials. $\quad 33 \quad$ (2012) 1281-1290. doi:10.1016/j.biomaterials.2011.10.067.

238. S. Naahidi, M. Jafari, M. Logan, Y. Wang, Y. Yuan, H. Bae B. Dixon, P. Chen, Biocompatibility of hydrogel-based scaffolds for tissue engineering applications, Biotechnol. $\begin{array}{llll}\text { Adv. } & 35 & \text { (2017) }\end{array}$ doi:10.1016/j.biotechadv.2017.05.006.

239. F. Song, X. Li, Q. Wang, L. Liao, C. Zhang, Nanocomposite hydrogels and their applications in drug delivery and tissue engineering, J. Biomed. Nanotech.. 11 (2015) 4052. doi:10.1166/jbn.2015.1962.

240. N. Bhattarai, J. Gunn, M. Zhang, Chitosan-based hydrogels for controlled, localized drug delivery, Adv. Drug Deliv. Rev. 62 (2010) 83-99. doi:10.1016/j.addr.2009.07.019.

241. Y. Wang, J. Wang, Z. Yuan, H. Han, T. Li, L. Li et al., Chitosan cross-linked poly(acrylic acid) hydrogels: Drug release control and mechanism, Colloids Surf. B Biointerfaces. $\quad 152 \quad$ (2017) 252-259. doi:10.1016/j.colsurfb.2017.01.008.

242. Ta, H., Dass, C. and Dunstan, D., 2008. Injectable chitosan hydrogels for localised cancer therapy. J. Control. Release, 126(3), pp.205-216.

243. H. Ta, C. Dass, D. Dunstan, Injectable chitosan hydrogels for localised cancer therapy, J. Control. Release. 126 (2008) 205-216. doi:10.1016/j.jconrel.2007.11.018.

244. H. Kang, H. Liu, X. Zhang, J. Yan, Z. Zhu, L. Peng, H. yang, Y. Kim, W. Tan, Photoresponsive DNA-Cross-Linked hydrogels for controllable release and cancer therapy, Langmuir. 27 (2011) 399-408. doi:10.1021/la1037553.

245. M. Sepantafar, R. Maheronnaghsh, H. Mohammadi, F. Radmanesh, M. Hasani-sadrabadi, M. Ebrahimi, H Baharvand, Engineered hydrogels in cancer therapy and diagnosis, Trends Biotechnol. 35 (2017) 1074-1087. doi:10.1016/j.tibtech.2017.06.015.

246. A. Hoffman, Hydrogels for biomedical applications, Adv. Drug Deliv. Rev. $64 \quad$ (2012) 18-23. doi:10.1016/j.addr.2012.09.010.

247. H. Abdeltawab, D. Svirskis, M. Sharma, Formulation strategies to modulate drug release from poloxamer based in situ gelling systems, Expert Opin. Drug Deliv. 17 (2020) 495-509. doi:10.1080/17425247.2020.1731469.

248. G. Giani, S. Fedi, R. Barbucci, Hybrid magnetic hydrogel: A potential system for controlled drug delivery by means of alternating magnetic fields, Polymers. 4 (2012) 1157-1169. doi:10.3390/polym4021157.

249. A. Paulino, A. Pereira, A. Fajardo, K. Erickson, M. Kipper, E. Muniz, L. Belfiore, E. Tambourgi, Natural polymerbased magnetic hydrogels: Potential vectors for remotecontrolled drug release, Carbohydrate Polymers. 90 (2012) 1216-1225. doi:10.1016/j.carbpol.2012.06.051.

250. S. Campbell, D. Maitland, T. Hoare, Enhanced pulsatile drug release from injectable magnetic hydrogels with embedded thermosensitive microgels, ACS Macro Lett. 4 (2015) 312-316. doi:10.1021/acsmacrolett.5b00057.

251.P. Ghasemiyeh, S. Mohammadi-Samani, Hydrogels as drug delivery systems; Pros and cons, Trends Pharm. Sci. 5 (2019) 7-24 doi: 10.30476/tips.2019.81604.1002
252.J. Lee, H. Oh, U. Baxa, S. Raghavan, R. Blumenthal, Biopolymer-connected liposome networks as injectable biomaterials capable of sustained local drug delivery, $\begin{array}{llll}\text { Biomacromolecules. } & 13 & \text { (2012) 3388-3394. }\end{array}$ doi:10.1021/bm301143d.

253. E. Ruel-Gariépy, G. Leclair, P. Hildgen, A. Gupta, J. Leroux, Thermosensitive chitosan-based hydrogel containing liposomes for the delivery of hydrophilic molecules, J. Control. Release. 82 (2002) 373-383. doi:10.1016/s0168-3659(02)00146-3.

254. A. Billard, L. Pourchet, S. Malaise, P. Alcouffe, A. Montembault, C. Ladavière, Liposome-loaded chitosan physical hydrogel: Toward a promising delayed-release biosystem, Carbohydr. Polym. 115 (2015) 651-657. doi:10.1016/j.carbpol.2014.08.120.

255. B. Ciobanu, A. Cadinoiu, M. Popa, J. Desbrières, C. Peptu, Modulated release from liposomes entrapped in chitosan/gelatin hydrogels, Mater. Sci. Eng. C. 43 (2014) 383-391. doi:10.1016/j.msec.2014.07.036.

256. S. Grijalvo, R. Eritja, D. Díaz, Liposomes-in-chitosan hydrogels: Challenges and opportunities for biomedical applications, Mater. Sci. Technol. (2019) 1-32. doi:10.1002/9783527603978.mst0484.

257. J. Dong, D. Jiang, Z. Wang, G. Wu, L. Miao, L. Huang, Intra-articular delivery of liposomal celecoxibhyaluronate combination for the treatment of osteoarthritis in rabbit model, Int. J. Pharm. 441 (2013) 285-290. doi:10.1016/j.ijpharm.2012.11.031.

258. S. Ren, Y. Dai, C. Li, Z. Qiu, X. Wang, F. Tian, S. Zhou, Q. Liu, H. Xing, Y. Lu, X. Chen, N. Li, Pharmacokinetics and pharmacodynamics evaluation of a thermosensitive chitosan based hydrogel containing liposomal doxorubicin, Eur. J. Pharm. Sci. 92 (2016) 137-145. Doi: 10.1016/j.ejps.2016.07.002

259. D. Cao, X. Zhang, M. Akabar, Y. Luo, H. Wu, X. Ke, T. Ci, Liposomal doxorubicin loaded PLGA-PEG-PLGA based thermogel for sustained local drug delivery for the treatment of breast cancer, Artif. Cells Nanomed. Biotechnol. 47 (2019) 181-191. Doi: 10.1080/21691401.2018.1548470

260.J. Dong, D. Jiang, Z. Wang, G. Wu, L. Miao, L. Huang, Intra-articular delivery of liposomal celecoxibhyaluronate combination for the treatment of osteoarthritis in rabbit model, Int. J. Pharm. 441 (2013) 285-290. Doi: 10.1016/j.ijpharm.2012.11.031

261.J. Lee, J. Gustin, T. Chen, G. Payne, S. Raghavan, Vesicle-biopolymer gels: Networks of surfactant vesicles connected by associating biopolymers, Langmuir. 21 (2005) 26-33. doi:10.1021/la048194+.

262. I. Kang, J. Yoon, Y. Lee, J. Choi, J. Yang, S. Chang, J. Lee, J. Lee, P. Yoo, J. Park. Stable vesicle assemblies on surfaces of hydrogel nanoparticles formed from a polysaccharide modified with lipid moieties, Chem. Eng. J. 263 (2015) 38-44. doi:10.1016/j.cej.2014.10.096.

263.S. Mourtas, S. Fotopoulou, S. Duraj, V. Sfika, C. Tsakiroglou, S. Antimisiaris, Liposomal drugs dispersed in hydrogels, Colloids Surf. B Biointerfaces. 55 (2007) 212-221. doi:10.1016/j.colsurfb.2006.12.005.

264.S. Mourtas, M. Haikou, M. Theodoropoulou, C. Tsakiroglou, S. Antimisiaris, The effect of added liposomes on the rheological properties of a hydrogel: A systematic study. J. Colloid Interface Sci 317 (2008) 611619. doi: 10.1016/j.jcis.2007.09.070

265. N. Lu, K. Yang, J. Li, Y. Weng, B. Yuan, Y. Ma, Controlled drug loading and release of a stimuli-responsive lipogel consisting of poly(N-isopropylacrylamide) particles and lipids, J. Phys. Chem. B. 117 (2013) 9677-9682. doi:10.1021/jp402826n.

266. Y. Wang, L. Chen, L. Tan, Q. Zhao, F. Luo, Y. Wei, Z. Qian, PEG-PCL based micelle hydrogels as oral docetaxel 
delivery systems for breast cancer therapy, Biomaterials. $\quad 35 \quad$ (2014) 6972-6985. doi:10.1016/j.biomaterials.2014.04.099.

267. O. Ivashkov, T. Yakimova, E. Evtushenko, A. Gelissen, F. Plamper, W. Richtering, A. Yaroslavov, On the mechanism of payload release from liposomes bound to temperature-sensitive microgel particles, Colloids Surf. A Physicochem. Eng. Asp. 570 (2019) 396-402. doi:10.1016/j.colsurfa.2019.02.069.

268. M. Faria, R. Machado, A. Ribeiro, H. Gonçalves, M. Real Oliveira, T. Viseu, J. Neves, M. Lúcio, Rational development of liposomal hydrogels: A strategy for topical vaginal antiretroviral drug delivery in the context of HIV prevention, Pharmaceutics. 11 (2019) 485. doi:10.3390/pharmaceutics11090485.

269. V. Trusova, K. Vus, U. Tarabara, O. Zhytniakivska, T. Deligeorgiev, G. Gorbenko, Liposomes integrated with amyloid hydrogels: a novel composite drug delivery platform, Bionanoscience. $10 \quad$ (2020) 446-454. doi:10.1007/s12668-020-00729-x.

270. E. Reimhult, Nanoparticle-triggered release from lipid membrane vesicles, New Biotechnol. 32 (2015) 665-672. doi:10.1016/j.nbt.2014.12.002.

271. K. Vargas, Y. Shon, Hybrid lipid-nanoparticle complexes for biomedical applications, J. Mater. Chem. B. 7 (2019) 695-708. doi:10.1039/c8tb03084g.

272. A. Wijaya, K. Hamad-Schifferli, High-density encapsulation of $\mathrm{Fe}_{3} \mathrm{O}_{4}$ nanoparticles in lipid vesicles, Langmuir. 23 (2007) 9546-9550. doi:10.1021/la701128b.

273. R. Mart, K. Liem, S. Webb, Creating functional vesicle assemblies from vesicles and nanoparticles, Pharm. Res. 26 (2009) 1701-1710. doi:10.1007/s11095-009-9880-8.

274.J. Haša, J. Hanuš, F. Štěpánek, Magnetically controlled liposome aggregates for on-demand release of reactive payloads, ACS Appl. Mater. Interfaces. 10 (2018) 2030620314. doi:10.1021/acsami.8b03891.

275. M. Ullrich, J. Hanuš, J. Dohnal, F. Štěpánek, Encapsulation stability and temperature-dependent release kinetics from hydrogel-immobilised liposomes, J. Colloid Interface Sci. 394 (2013) 380-385. doi:10.1016/j.jcis.2012.11.016.

276. R. Mart, K. Liem, S. Webb, Magnetically-controlled release from hydrogel-supported vesicle assemblies, Chem. Commun. (2009) 2287-2289. doi:10.1039/b901472a.

277. F. de Cogan, A. Booth, J. Gough, S. Webb, Conversion of magnetic impulses into cellular responses by selfassembled nanoparticle-vesicle hydrogels, Angew. Chem. Int. Ed. 123 (2011) 12498-12501. doi:10.1002/ange.201103469.

278. F. de Cogan, A. Booth, J. Gough, S. Webb, Spatially controlled apoptosis induced by released nickel(II) within a magnetically responsive nanostructured biomaterial, Soft Matter. 9 (2013) 2245-2253. doi:10.1039/c2sm27379a.

279. R. Robert Blumenthal, Magnetically triggered drug release from liposome embedded gel, J. Nanomed. Biotherapeutic Discov. 4 (2014) 1000130. doi:10.4172/2155-983x.1000130.

280.J. Hanuš, M. Ullrich, J. Dohnal, M. Singh, F. Štěpánek, Remotely controlled diffusion from magnetic liposome microgels, Langmuir. $29 \quad$ (2013) 4381-4387. doi:10.1021/la4000318.

281. A. Pittermannová, Z. Ruberová, A. Zadražil, N. Bremond, J. Bibette, F. Štěpánek, Microfluidic fabrication of composite hydrogel microparticles in the size range of blood cells, RSC Adv. 6 (2016) 103532-103540. doi:10.1039/c6ra23003b.

282. M. van Elk, C. Lorenzato, B. Ozbakir, C. Oerlemans, G. Storm, F. Nijsen, R. Deckers, T. Vermonden, W. Hennink, Alginate microgels loaded with temperature sensitive liposomes for magnetic resonance imageable drug release and microgel visualization, Eur. Polym. J. 72 (2015) 620-631. doi:10.1016/j.eurpolymj.2015.03.013.

283. M. Ullrich, J. Hanuš, F. Štěpánek, Remote control of enzymatic reaction in compartmentalized microparticles: A system for the delivery of unstable actives, Chem. Eng. Sci. 125 (2015) 191-199. doi:10.1016/j.ces.2014.06.020.

284. D. Kang, H. Jung, N. Ahn, S. Yang, S. Seo, K. Suh, P. Chang, N. Jeon, J. Kim, K. Kim, Janus-compartmental alginate microbeads having polydiacetylene liposomes and magnetic nanoparticles for visual lead(II) detection, ACS Appl. Mater. Interfaces. 6 (2014) 10631-10637. doi:10.1021/am502319m.

285. R. Das, N. Rinaldi-Montes, J. Alonso, Z. Amghouz, E. Garaio, J. García, P. Gorria, J. Blanco, M. Phan, H. Srikanth, Boosted hyperthermia therapy by combined ac magnetic and photothermal exposures in $\mathrm{Ag} / \mathrm{Fe} 3 \mathrm{O} 4$ nanoflowers, ACS Appl. Mater. Interfaces 8 (2016) 25162-25169.

286. M. Kwak, I. Jung, Y. Kang, D. Lee, S. Park, Multi-block magnetic nanorods for controlled drug release modulated by Fourier transform surface plasmon resonance, Nanoscale 10 (2018) 18690-18695.

287. Y. Jin, C. Jia, S. Huang, M. O’Donnell, X. Gao, 2010. Multifunctional nanoparticles as coupled contrast agents, Nat. Commun. 1, 1395.

288. T. Stewart, A. Nagesetti, R. Guduru, P. Liang, E., Stimphil, A. Hadjikhani, L. Salgueiro, J. Horstmyer, R. Cai, A. Schally, S. Khizroev, Magnetoelectric nanoparticles for delivery of antitumour peptides into glioblastoma cells by magnetic fields, Nanomedicine 13 (2018) 423-438. 